\title{
Qing-Luo-Yin Alleviated Experimental Arthritis in Rats by Disrupting Immune Feedback Between Inflammatory T Cells and Monocytes: Key Evidences from Its Effects on Immune Cell Phenotypes
}

\author{
Dan-Dan Wang ${ }^{1,2, *}$ \\ Xin-Yue Wu (iD) ${ }^{3, *}$ \\ Ji-Yang Dong (D) ${ }^{3}$ \\ Xiu-Ping Cheng ${ }^{1,2}$ \\ Shao-Fei Gu ${ }^{1,2}$ \\ Opeyemi Joshua Olatunji (iD) ${ }^{4}$ \\ Yan $\mathrm{Li}^{1,2}$ \\ Jian Zuo ${ }^{1,5,6}$ \\ 'Xin'an Medicine Research Center, The \\ First Affiliated Hospital of Wannan \\ Medical College (Yijishan Hospital), \\ Wuhu, 241000, People's Republic of \\ China; ${ }^{2}$ Research Center of Integration of \\ Traditional Chinese and Western \\ Medicine, Wannan Medical College, \\ Wuhu, 241000, People's Republic of \\ China; ${ }^{3}$ Department of Electronic \\ Science, Xiamen University, Xiamen, \\ 361005, People's Republic of China; \\ ${ }^{4}$ Faculty of Traditional Thai Medicine, \\ Prince of Songkla University, Hat Yai, \\ 901 10, Thailand; ${ }^{5}$ Key Laboratory of \\ Non-Coding RNA Transformation \\ Research of Anhui Higher Education \\ Institution, Wannan Medical College, \\ Wuhu, 241000, People's Republic of \\ China; ${ }^{6}$ Anhui Provincial Engineering \\ Laboratory for Screening and Re- \\ Evaluation of Active Compounds of \\ Herbal Medicines in Southern Anhui, \\ Wuhu, 241000, People's Republic of \\ China
}

*These authors contributed equally to this work

Correspondence: Yan Li; Jian Zuo Email liyan.0301@163.com; zuojian8I78@I63.com
Background: Qing-Luo-Yin (QLY) is an anti-rheumatic herbal formula. Despite the well-investigated therapeutic efficacy of QLY, its immune regulatory properties are largely unknown. $\mathrm{CD}^{+} \mathrm{T}$ cells and monocytes are two key parameters in rheumatoid arthritis (RA). This study investigated the changes in these cells in QLY-treated RA animal models.

Materials and Methods: RA models were induced in male SD rats and were orally treated with QLY. Dynamic metabolic changes in collagen-induced arthritis (CIA) rats were monitored by ${ }^{1} \mathrm{H}$ NMR approach. The immunity profiles of CIA and adjuvantinduced arthritis (AIA) rats were evaluated using immunohistochemical, PCR, ELISA, cytokine chip, flow cytometry, and immunofluorescence experiments. The bioactive components in QLY were identified by bioinformatic-guided LC-MS analyses. The compounds with high abundance in QLY decoction and easily absorbed were taken as key anti-rheumatic components and used to treat blood-derived immune cells using in vitro experiments.

Results: The results indicated that QLY decreased Th17 cells frequency and T cellsreleased IL-6, IL-17 and GM-CSF in CIA rats, which was attributed to the impaired lymphocyte maturation and altered differentiation. QLY inhibited lactic acid production and inflammatory polarization in the monocytes during the peak period of AIA and CIA. AIA monocytes elicited significant increase in Th17 cells counts, IL-6 and IL-1 $\beta$ secretion in co-cultured splenocytes, which was abrogated by QLY. QLY-containing serum suppressed the phosphorylation of JNK and p65 in AIA lymphocyte-stimulated normal monocytes and consequently inhibited iNOS and IL-1 $\beta$ expression as well as IL-6 and IL-1 $\beta$ production. Matrine, sinomenine and sophocarpine were identified as major bioactive compounds in QLY. These identified compounds effectively inhibited the development of inflammatory $\mathrm{T}$ cells using concentrations detected in QLY-treated rats. At higher concentrations (20-fold increase), the chemical stimuli significantly suppressed the production of IL-1 $\beta$ in AIA monocytes by inhibiting JNK and p65 pathways.

Conclusion: By targeting inflammatory T cells and monocytes as well as disrupting their interplay, QLY improved immune environment in RA models especially during the active stages of disease.

Keywords: traditional Chinese medicine, rheumatoid arthritis, innate immunity, $\mathrm{T}$ cells, chronic inflammation, metabolomics 


\section{Introduction}

In spite of the significant advancement in medical science in the past decades, the successful treatment of rheumatoid arthritis (RA) is still a big challenge. It is a consensus nowadays that RA is mainly driven by $\mathrm{CD} 4^{+} \mathrm{T}$ cells. ${ }^{1}$ The expansion of autoreactive $\mathrm{T}$ cells occurs several years before the clinical manifestation of RA. ${ }^{1,2}$ It serves as an important indicator of self-tolerance breakdown, and it also positively correlates to arthritic manifestations. ${ }^{2}$ Infections, major histocompatibility complex gene polymorphism, environmental pollution, and unhealthy life habits can all possibly initiate this abnormality. ${ }^{3}$ Through excretion, autoreactive $\mathrm{T}$ cells reshape the immune milieu of RA patients. ${ }^{2,3}$ As a result, $\mathrm{T}$ cells are dominantly differentiated into inflammatory phenotypes. The population of Th1 and Th17 cells are significantly expanded, while the development of Th2 and Treg cells is impaired. ${ }^{1,3}$ Initially, Th1 cells were thought to situate in the center of RA pathology because its inflammatory nature is well accepted and high levels of IFN- $\gamma$ are observed in RA patients. ${ }^{4}$ Later, Th17 cells was revealed as an equally important player in RA pathogenesis. ${ }^{5}$ Consequently, restoring the balance of $\mathrm{T}$ cell subsets and cytokine network is deemed as the most promising antirheumatic strategy, which has inspired the successful development of many therapeutic RA drugs. ${ }^{4-6}$ However, as a part of the adaptive immunity, $\mathrm{T}$ cells do not function independently. Their interaction with innate immune system is inevitable and important.

Innate immunity is crucial for host defense especially during the early stages of infection, and required for lymphocyte maturation. The relevant immune cells typically have the ability to eliminate pathogens and present antigens. The dysfunction of monocytes, the major component of innate immunity is deeply implicated in RA mainly through their interplay with $\mathrm{T}$ cells. ${ }^{7}$ Polarization profile of monocytes in RA patients is largely different from healthy subjects and the frequency of inflammatory $\mathrm{CD} 16^{+}$monocytes is obviously increased in RA patient, which positively correlates to the severity of the disease. ${ }^{8,9}$ More importantly, $\mathrm{CD} 14^{++} \mathrm{CD} 16^{+}$monocytes can directly promote Th17 differentiation. ${ }^{7,9}$ Due to the different surface molecule composition, CD14/CD16 classification criterion failed to define monocyte subsets in rodents. ${ }^{10,11}$ Nonetheless, inflammatory monocytes characterized by other molecular phenotypes similarly contribute to the pathological changes in rodent rheumatic models. ${ }^{12}$
Regardless of species differences, inflammatory monocytes efficiently generate inflammatory cytokines, chemokines, matrix metalloproteinases and reactive oxygen species, and consequently affect $\mathrm{T}$ cell differentiation. ${ }^{12,13}$ We recently discovered that inflammatory polarization of monocytes coincides to the onset of polyarthritis during the course of collagen-induced arthritis (CIA) (unpublished data). It is reasonable to deduce that even though restoring $\mathrm{CD}^{+} \mathrm{T}$ cells homeostasis is a priority for RA treatment, monocytes-targeting therapies could be similarly effective in treating inflammatory symptoms. ${ }^{13}$

Conventional disease modifying anti-rheumatic drugs (cDMARDs) are useful in easing arthritic symptoms, but they barely affect the long-term prognosis of RA. ${ }^{14}$ As a representative cDMARD, methotrexate (MTX) is widely used as one of the first-line drugs in RA therapies. Low dose MTX regimen exerts good anti-inflammatory effects by activating adenosine receptor, but majority of RA patients do not respond to it very well. ${ }^{15,16}$ Comparatively, biological DMARDs (bDMARDs) have obvious therapeutic advantages. Accumulating knowledge about RA immunity empowers the excellent antirheumatic effects of these drugs. ${ }^{6}$ However, their efficacy could be limited in clinical practices, because RA etiology is heterogeneous, and this disease is controlled by the whole immune network rather than certain exclusive factors. ${ }^{17,18}$ The blocking antibody of tumor necrosis factor alpha (TNF- $\alpha$ ) etanercept is a good example. Theoretically, it can substantially improve immune environment, because TNF- $\alpha$ increase is a key event of RArelated cytokine network disturbance. ${ }^{3,19}$ Undoubtedly, its long-term use affords tangible benefits to patients, but the rate of significant improvement is only about $20 \%{ }^{19}$ The development of multi-target drugs and combined use of bDMARDs could be possible solutions; however, these measures are not feasible in the near future, considering the safety concerns and tremendous uncertainties. ${ }^{20,21}$

Alternative therapies have provided another possible therapeutic option. Herbal formula contains numerous ingredients and their combination may be more effective in treating the multi-facets nature of RA. ${ }^{22}$ Qing-Luo-Yin (QLY) is a representative formula of $\mathrm{Ji}-\mathrm{Ren} \mathrm{Li}$, a famous contemporary traditional Chinese medicine (TCM) master. It has been successfully used in RA treatments for over 40 years. ${ }^{23}$ This formula is specifically designed to treat hot syndromes-related RA, and it is composed of four herbal ingredients: Radix Sophorae flavescentis (Ku Shen, KS), 
Caulis Sinomenium acutum (Qing Feng Teng, QFT), Cortex Phellodendri chinensis (Huang Bo, HB) and Rhizoma Dioscoreae hypoglaucae (Bi Xie, BX). As the principal herbal component, KS accounts for the general therapeutic properties of QLY. The assistant drug QFT, HB and $\mathrm{HB}$ reinforces its effects by expelling pathogenic dampness and heat. From phytochemical perspective, KS, HB and QFT are all rich in alkaloids. ${ }^{24-26}$ Therefore, alkaloid derivatives are usually taken as key bioactive components within QLY. ${ }^{27}$ The current study demonstrated that QLY can simultaneously target inflammatory T cells and monocytes, and its alkaloids significantly contributed to the therapeutic effects of QLY on CIA and adjuvantinduced arthritis (AIA). It should be noted that the pathogenesis and clinical manifestations of CIA and AIA are largely different. T cells-governed autoimmune reactions in CIA models are notable, while monocytes-mediated inflammation is the dominant clinical manifestation of AIA. ${ }^{28}$ Evidences from the two models are necessary to draw a solid conclusion.

\section{Materials and Methods Materials and Reagents}

Lyophilized immunization grade bovine type II collagen (CII, 20021, purified from bovine articular cartilage, with the purity $>99 \%$ by SDS-PAGE, free of pepsin and proteoglycans) and incomplete Freund's adjuvant (IFA, 7002) were obtained from Chondrex Inc. (Redmond, WA, USA). Rat enzyme-linked immunosorbent assay (ELISA) kits for the quantification of granulocyte-macrophage colony stimulating factor (GM-CSF, 70-EK363), interleukin 1 beta (IL-1 $\beta$, 70-EK301B/3), interleukin 17 alpha (IL-17 $\alpha, 70-E K 317 / 3$ ), TNF- $\alpha$ (70-EK382/3) and interleukin 6 (IL-6, 70-EK306/3) together with phorbol 12-myristate 13-acetate (PMA, CS1001) and brefeldin A (BFA, CS1002) were purchased from MultiScience Biotech (Hangzhou, Zhejiang, China). Heat-inactivated Bacillus Calmette-Guérin (BCG, R19021), TRNzol Universal reagent (DP424), Universal qPCR Master Mix (M3003L) and fetal bovine serum (FBS, S711-001S) were supplied by Rebio Scientific (Shanghai, China), Tiangen Biotech (Beijing, China), New England Biolabs (Ipswich, MA, USA) and Lonza Science (Shanghai, China), respectively. Anti-rat primary p65 (A19653), JNK (A11119), p-JNK (AP0473) antibodies were obtained from ABclonal Technology (Wuhan, Hubei, China). Anti-rat primary p-p65 (AF3387) and p-STAT3 (AF3294) antibodies together with anti-mouse horseradish peroxidase (HRP)- linked secondary antibody (S0002) were provided by Affinity Biosciences (Changzhou, Jiangsu, China). Anti-rat primary CD43 (Sc-53044), SIRT1 (D107) and STAT3 (10253-2-AP) antibodies were purchased from Santa Cruz Biotechnology (Santa Cruz, CA, USA), Cell Signaling Technology (Danvers, MA, USA) and Proteintech Group (Chicago, IL, USA), respectively. Red donkey anti-rabbit (A24421) and orange donkey anti-mouse (A24311) secondary antibodies were bought from Abbkine (Wuhan, Hubei, China). Anti-rat FITC-CD3 (11-0030-82), PE-CD4 (120040-82), APC-IL-17 $\alpha \quad$ (17-7177-81), FITC-CD172a (MA1-90103), PE-CD43 (MA5-17386), PE-CD86 (120860-83) and FITC-CD11b (MA5-17509) antibodies, eBioscience $^{\mathrm{TM}}$ Fixation/Permeabilization Concentrate $(00-$ 5123-43), ReverAid First Strand cDNA Synthesis kit (K1622) and RPMI-1640 medium (SH30809.1) were obtained from Thermo Fisher Scientific (Rockford, IL, USA). Anti-rabbit HRP-linked secondary antibody (BL003A) and red cell lysis buffer (BL503B) were supplied by Biosharp Life Science (Hefei, Anhui, China). BCA kit (P0012S), penicillin-streptomycin (C0222), RIPA lysis buffer (P0013B), SDS-PAGE sample loading buffer (P0015L), antifade mounting medium (P0126) and QuickBlock ${ }^{\mathrm{TM}}$ blocking buffer (P0260) were purchased from Beyotime Biotechnology (Shanghai, China). Rat peripheral blood lymphocyte isolation kit (P8630) and rat peripheral blood monocyte isolation kit (P6700) were procured from Solarbio (Beijing, China). Sinomenine (SIN, B20997), sophocarpine (SCA, B20544) and matrine (MT, B20679) were purchased from Yuanye Bio-Technology (Shanghai, China). The reagents used in NMR analysis were of spectrographic grade. Information about other reagents used together with all the lot numbers is included in Supplementary S1.

\section{Preparation of QLY Extract}

The raw herbal materials $\mathrm{KS}, \mathrm{QFT}, \mathrm{HB}$ and $\mathrm{BX}$ were purchased from Bozhou Herbal Medicine Market (Anhui, China) and were identified by Assoc Professor Jian Zuo (Wannan Medical College, Anhui, China). These ingredients were mixed at the ratio of 1.5:1.2:1:1 and powdered. The powdered mixture was refluxed with 95\% ethanol twice, and the remaining residues were further decocted in boiling water twice. The combined filtrates was dried to obtain a sticky extract, which was then diluted with normal saline to obtain a suspension at the concentration of $0.5 \mathrm{~g} /$ $\mathrm{mL}$ for further use. 


\section{General Design of in vivo Experiments}

Rats show no obvious gender difference in susceptibility to rheumatic models. ${ }^{29}$ To simplify the experiments, both CIA and AIA models were induced in male SpragueDawley rats (7 weeks old), which were supplied by Tianqin Biotechnology (Changsha, Hunan, China). Compared to AIA, the pathogenesis of CIA is more similar to RA. CIA is characterized by hyperactivation of $\mathrm{CD}^{+}$ $\mathrm{T}$ cells and high autoreactivity, while monocytes-driven inflammation is more obvious in AIA. ${ }^{28}$ To better characterize immune consequences from QLY treatment, we firstly investigated the changes in treated CIA rats, and the results obtained were further tested using AIA models. All the animal experimental protocols were performed in accordance with the National Institutes of Health Guide for the Care and Use of Laboratory Animals (NIH Publications No. 8023, revised 1978) and approved by the Ethics Committee of Wannan Medical College (LLSC-2020-138). The rats were adaptively fed for 7 days beforehand, and allowed ad libitum access to food and water. The temperature, relative humidity, and circadian rhythm were set at $24 \pm 2^{\circ} \mathrm{C}, 50 \pm 2 \%$, and $12 \mathrm{~h}$ per cycle (8:00-20:00), respectively. The induction of CIA and AIA models was according to the protocols provided by Chondrex Inc.

\section{CIA Induction, Treatments and Sampling}

CII was dissolved in $0.05 \mathrm{M}$ acetic acid one day before induction of CIA, and placed overnight at $4^{\circ} \mathrm{C}$. The CII solution $(2 \mathrm{mg} / \mathrm{mL})$ was then slowly mixed with the same volume of IFA by stirring until a stable emulsion was obtained. A subcutaneous injection of this emulsion $(0.1 \mathrm{~mL})$ was performed on tail base with a glass Hamilton syringe, and the day was recorded as day 0 . Thereafter, 12 CIA induced rats were equally assigned into QLY treatment and CIA model groups. QLY-treated CIA rats received an oral dose of QLY $(0.45 \mathrm{~g} / \mathrm{kg}$, relative to the dry extract) daily for 37 days. The dose volume for each rat was approximately $3 \mathrm{~mL}$ according to the body weight. CIA models and another 6 healthy rats were treated with $3 \mathrm{~mL}$ of normal saline in parallel.

Polyarthritis usually develops two weeks after CII injection in rats, and obvious inflammation approximately lasts for 20 days. To cover the whole inflammatory duration, we collected blood samples from the fossa orbitalis vein on day 14, 21, 28 and 35 since the presumed onset of arthritis to investigate the dynamic changes in immune status under QLY treatment. The serum was divided into aliquots for different analytical purposes. On day 38, all the rats were sacrificed and blood was collected through abdominal aorta. The thymus and hind limbs including paws were dissected, and immediately immersed in neutral formalin buffer. The hind leg bones from healthy rats were separated from the hip joint and placed in a culture dish containing culture medium. The epiphysis of the separated femur and tibia was cut off at both ends and the bone marrow was flushed out with RPMI 1640 medium with a syringe.

\section{Analyses of Biological Samples from CIA Rats}

The levels of GM-CSF, IL- 6 and IL-17 $\alpha$ in periodically collected serum were determined by corresponding ELISA kits. The remaining analyses were performed on samples obtained upon the sacrifice of the rats. The levels of IL-1 $\beta$ and TNF- $\alpha$ in serum were similarly detected using ELISA kits. Serological cytokine composition was investigated using a cytokine chip. Every three samples from a group were mixed as a pooled analyte, and the levels of 67 cytokines were determined using a Quantibody ${ }^{\circledR}$ Array Glass Chip (Cat No. GSR-CAA-67). Both the chip and testing service were provided by Raybiotech (Guangzhou, Guangdong, China). The anticoagulant blood samples were subjected to either complete blood cell counts on a PE-6800 VIT blood cell counter (Pukang Biotech, Shenzhen, Guangdong, China) or flow cytometry analysis with procedures detailed below.

Histological and immunohistochemical experiments were performed according to procedures reported previously. ${ }^{30}$ Decalcified joints (in 10\% ethylenediamine tetraacetic acid) and fixed thymus were embedded in paraffin, and sectioned at $5 \mu \mathrm{m}$. Some of the joint slices were dyed with hematoxylin and eosin (H\&E) for general histological examination. The remaining joint and thymus sections were dewaxed with xylene, and re-hydrated in PBS. The endogenous peroxidase was inactivated using $3 \%$ hydrogen peroxide, and antigen repair was achieved using citric acid microwave heating method. After being blocked with normal goat serum for $30 \mathrm{~min}$ at $37^{\circ} \mathrm{C}$, the sections were treated with rabbit anti-rat primary polyclonal antibodies $(1: 100)$ at $4{ }^{\circ} \mathrm{C}$ overnight, and further incubated with goat anti-rabbit biotin-conjugated secondary antibody (1:2000). Signals were developed with HRP- 
labeled streptavidin and 3,3'-diaminobenzidine, and hematoxylin staining was performed.

Fresh bone marrow was firstly inculcated with red cell lysis solution under room temperature for $10 \mathrm{~min}$. After centrifugation, the remaining cells were collected and seeded in 6 -wells plates $\left(1 \times 10^{6}\right.$ cells/well $)$. The cells were then incubated in $0.9 \mathrm{~mL}$ of RPMI-1640 medium supplemented with $10 \%$ FBS under normal culture conditions. QLY extract was dried in vacuum and the residue was dissolved in water-ethanol (70:30) to obtain a stock solution $(15 \mathrm{mg} / \mathrm{mL})$, which was then diluted with PBS to obtain a solution at $300 \mu \mathrm{g} / \mathrm{mL}$. After $2 \mathrm{~h}$ incubation, $0.1 \mathrm{~mL}$ of filtered QLY solution $(0.22 \mu \mathrm{m})$ was added. The culture lasted for $12 \mathrm{~h}$. The cells were finally harvested for real-time quantitative PCR (RT-qPCR) analysis.

\section{NMR Analysis of Metabolites in Serum}

To characterize the dynamic metabolic changes of rats, periodically collected serum was subjected to ${ }^{1} \mathrm{H}$ NMR analysis. An aliquot of $150 \mu \mathrm{L}$ of the serum was diluted with $\mathrm{D}_{2} \mathrm{O}$-based PBS. After centrifugation, $450 \mu \mathrm{L}$ of the supernatant was transferred to 5-mm NMR tubes. ${ }^{1} \mathrm{H}$ NMR spectra was acquired on a Bruker Advance Spectrometer (Bruker BioSpin, Karlsruhe, Germany) operating at 850.29 $\mathrm{MHz}$ and $298 \mathrm{~K}$ temperature. Spectra processing was performed on MestReNova v.14.1.1 (Mestrelab Research S.L., La Coruña, Spain). After Fourier transformation and manual baseline correction, the left split of the doublet from lactic acid was set at $1.336 \mathrm{ppm}$ as a reference for chemical shift correction. The residual water signals, urea resonances and peak-free regions were selectively excluded, while the remaining spectra were segmented into bucketed data based on self-adaptive integration, and the results were exported as xlsx files. The date was normalized using probabilistic quotient normalization (PQN) method to compensate for overall concentration variations. The peaks were subsequently assigned to different metabolites based on previously published data. ${ }^{31}$ Their relative abundance was assessed based on normalized peak integral area. For the metabolites that gave rise to multiple peaks, peaks in the least overlapping region were chosen for quantification. Partial least squaresdiscrimination analysis (PLS-DA) and corresponding seven-fold cross-validation were performed using SIMCA v.14.1 (Umetrics, Umeå, Sweden). Variable importance in projection (VIP) was used to evaluate the contribution of variables to the model of PLS-DA. Its detailed definition and calculation can be found in a previous report. ${ }^{32}$ The differential metabolites identified were then ranked based on VIP values, which indicated their discriminatory potentials.

\section{AIA Induction, Treatments and Immune Profile Characterization}

IFA and BCG were finely grinded to prepare complete Freund's adjuvant (CFA, containing $20 \mathrm{mg} / \mathrm{mL}$ of BCG). Twelve rats received a subcutaneous injection of $0.1 \mathrm{~mL}$ of CFA at the left hind paw pad. The injected rats were equally divided into AIA model and QLY-treated groups. Another six rats served as healthy controls. The treatments were the same with those depicted above. On day 18 , peripheral blood was collected. The distribution of monocyte and $\mathrm{T}$ cell subsets was investigated by flow cytometry. After erythrolysis, white blood cells (WBCs) were obtained and the mRNA expression of iNOS, IL-1 $\beta$, MCP-1, IL-10, Arg-1 and SIRT1 in the WBCs was evaluated using RT-qPCR. Co-localization of protein CD43 and SIRT1 in peripheral monocytes was observed by immunofluorescence method. On day 28, all the rats were killed, and blood was collected for subsequent in vitro experiments. Lymphocytes and monocytes from blood were prepared using corresponding isolation kits.

\section{Co-Treatment of Immune Cells from AIA Rats}

In the co-culture experiments, we firstly assessed the influence of different rats-derived monocytes on normal $\mathrm{T}$ cells. Because the distribution of $\mathrm{T}$ cells is limited in blood, splenocytes were used in this assay. A fresh spleen dissected from healthy rat was carefully grinded under sterile conditions. The resulting cellular suspension was filtered through 200 mesh, and the splenocytes were obtained after erythrolysis. Normal splenocytes were subsequently co-cultured with blood monocytes obtained from different rats. Splenocytes were located in the upper chamber of the transwell $(0.4 \mu \mathrm{m})$, and the monocytes were seeded in the lower chamber. The cell density of all the coculture assays was $1 \times 10^{6}$ cells/well. After $12 \mathrm{~h}$, the splenocytes were harvested for flow cytometry analysis, and levels of IL- 6 and IL- $1 \beta$ in medium from the upper chambers were analyzed using ELISA kits.

Thereafter, we attempted to clarify if QLY treatment alleviated monocytes-mediated inflammation by targeting lymphocytes in AIA rats. To better simulate physiological conditions, cells were stimulated with QLY-containing 
serum rather than herbal extract. A healthy rat was given three consecutive oral doses of QLY at intervals of 1 h. One hour after the last administration, the rat together with another untreated rat were sacrificed and maximum amount of blood was collected for serum preparation. The lymphocytes collected from healthy or AIA rats were incubated in the upper chamber and pre-exposed to different rat serum (10\%) for $2 \mathrm{~h}$. Subsequently, normal monocytes were seeded in the lower chamber and received no extra stimulus. After $12 \mathrm{~h}$ incubation, the medium from lower chambers was used for ELISA analysis, and the monocytes were collected for PCR and western-blot (WB) experiments.

\section{Identification of Bioactive Components in QLY}

In this assay, we firstly predicted the anti-rheumatic mechanism of QLY using a network pharmacology method. The obtained results were then tested by experimental clues. Afterwards, the compounds contributing significantly to the anti-rheumatic properties of QLY were identified. However, network pharmacological analysis is qualitative. Even though a compound targets multiple genes, it cannot be solidly concluded that it is a key component. Effective concentration is another decisive variation. Hence, among the bioactive compounds, only those abundant in QLY decoction and easily absorbed were taken as key bioactive components.

Chemical structures and reported targets of QLYrelated bioactive compounds were downloaded from traditional Chinese medicine Systems Pharmacology Database and Analysis Platform (https://old.tcmsp-e.com/tcmsp. php). All possibly regulated genes were enriched in pathways with the aid of public bioinformatics libraries KEGG (Kyoto Encyclopedia of Genes and Genomes) and GO (Gene Ontology). Based on the results, key pathways related to anti-rheumatic properties of QLY were screened out, and the interactions between bioactive compounds and corresponding genes were visualized using Cytoscape software. $^{33}$

In the LC-MS experiments, supernatant from methanol-denatured serum and diluted decoction was dried in the vacuum, and the analytes were re-constructed using methanol. After filtration $(0.22 \mu \mathrm{m})$, samples were injected into a Dionex UltiMate UPLC (Thermo Fisher Scientific) for chromatography separation using a gradient elution mobile phase comprising $0.1 \%$ formic acid in water and
$0.1 \%$ formic acid in acetonitrile at $0.4 \mathrm{~mL} / \mathrm{min}$. Ion fragments were detected by a Q Exactive Focus mass spectrometer (Thermo Fisher Scientific) under Full Scan-ddMS ${ }^{2}$ mode. The main detection parameters include sheath gas flow rate, 45 Arb; aux gas flow rate, 15 Arb; capillary temperature, $400^{\circ} \mathrm{C}$, full $\mathrm{ms}$ resolution, 70,000 , MS/MS resolution, 17,500, collision energy, 15/30/45 in NCE mode; spray voltage, $4.0 \mathrm{kV}$ (positive) or $-3.6 \mathrm{kV}$ (negative); mass range, 100 to 1500 . The compounds were identified by searching an in-house constructed natural compound $\mathrm{MS}^{2}$ database (Biotree Biotech, Shanghai, China), and their abundance was assessed according to the relative signal intensity normalized by internal reference 2-chloro-1-phenylalanine.

Afterwards, we accurately quantified key components in QLY-containing serum using the platform comprising a 1260 Infinity UPLC and a 6460 triple-quadrupole mass spectrometer (Agilent Technology MA, USA). The analytes were separated on an Agilent $\mathrm{C} 18$ column $(2.1 \times 50 \mathrm{~mm}, 1.8 \mu \mathrm{m})$. The mobile phase was comprised of methanol and $0.1 \%$ formic acid in water $(20: 80)$, and flow rate was set at $0.3 \mathrm{~mL} / \mathrm{min}$. The injection volume and total run time were $5 \mu \mathrm{L}$ and $4 \mathrm{~min}$, respectively. The column was equilibrated for $5 \mathrm{~min}$ before each injection. The determination was performed under positive electrospray ionization mode with following mass spectrometry parameters: fragmentor voltages, $135 \mathrm{~V}$; collision energy, $15 \mathrm{eV}$; gas temperature, $300^{\circ} \mathrm{C}$; gas flow, $5 \mathrm{~L} / \mathrm{min}$; nebulizer gas, $45 \mathrm{psi}$; sheath gas temperature, $250^{\circ} \mathrm{C}$; sheath gas flow, $11 \mathrm{~L} / \mathrm{min}$; capillary voltage, $3500 \mathrm{~V}$. The quantification of compounds was achieved on Multiple Reaction Monitoring (MRM) mode. Parent/daughter ions $(\mathrm{m} / \mathrm{z})$ were as follows: $330 / 181.2$ for SIN; $249.3 / 148.1$ for MT; 247/96 for SCA.

\section{Treatment of Immune Cells with Bioactive Compounds}

In this experiment, we stimulated lymphocytes/monocytes directly with bioactive compounds identified above. Immune cells derived from different rats were seeded in 6 -wells plates at a density of $1 \times 10^{6}$ cells/well. After adaptive culture, the attached cells were treated with the compounds separately or in combination for $12 \mathrm{~h}$, and then harvested after washing with ice-cold PBS. The mRNA expression of SIRT1, Arg-1, IL-10, iNOS, IL-1 $\beta$ IL-4, IFN- $\gamma$ and IL-17 $\alpha$ in the cells were evaluated using RTqPCR, and the protein expression of (p)-p65, (p)-JNK, (p)- 
STAT3 were assessed by WB method. The levels of IL- $1 \beta$, IL-6, IFN- $\gamma$ and IL-17 $\alpha$ in medium were quantified using ELISA kits.

\section{ELISA Tests}

The supernatant from the cell culture medium and serum were collected after centrifugation. Primary antibodycoated plates were soaked in $300 \mu \mathrm{L}$ washing solution for $30 \mathrm{~s}$ beforehand. Thereafter, $100 \mu \mathrm{L}$ of the diluted samples or standard solutions were added into each well, which was followed by incubation at $37^{\circ} \mathrm{C}$ for $1 \mathrm{~h}$. After that, the liquid was replaced with $100 \mu \mathrm{L}$ detection antibody solution, and the plates were further incubated for 2 h. All the following incubations were performed at room temperature on a shaker (300 rpm). Subsequently, the detection antibody was discarded, and the plates were incubated with HRP-labeled streptavidin (100 $\mu \mathrm{L} /$ well)

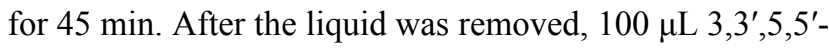
tetramethylbenzidine solution was added and incubated for $20 \mathrm{~min}$. The plates were extensively rinsed at every interval. Finally, $100 \mu \mathrm{L}$ termination solution was used to stop the reaction. The optical density was read at $450 \mathrm{~nm}$ by taking $570 \mathrm{~nm}$ as the reference wavelength.

\section{Flow Cytometry Analyses}

The cells obtained from the experiments stated above were firstly re-suspended in flow cytometry staining buffer. To analyze $\mathrm{T}$ cell subsets, the cells were treated with PMA and BFA for $6 \mathrm{~h}$ to augment cytokine secretion and block extracellular transportation. The processed cells were incubated with anti-rat FITC-CD3 and PE-CD4 antibodies at room temperature for 15 min in dark, and then treated with eBioscience ${ }^{\mathrm{TM}}$ Fixation/Permeabilization Concentrate. Intracellular IL$17 \alpha$ was tagged with anti-rat APC-IL-17 $\alpha$ antibody with further incubation for $20 \mathrm{~min}$. After filtration, the unicellular suspension was fed to a FACSVerse Flow Cytometer (BD Biosciences, San Jose, CA, USA), and $1 \times 10^{4}$ cells were acquired each time. Monocytes were directly dyed with the combination of fluorescein-tagged anti-rat CD86-CD11b or CD43-CD172 antibodies. Other procedures were the same with those depicted above.

\section{Immunofluorescence Experiment}

Peripheral blood mononuclear cells were maintained in complete culture medium in confocal dishes immediately after isolation. Unattached lymphocytes were removed, and the remaining monocytes were fixed and permeabilized with appropriate buffers, which was followed by blocking procedure using QuickBlock ${ }^{\mathrm{TM}}$ immu- $^{\mathrm{m}}$ nostaining blocking buffer. After that, primary rabbit antirat SIRT1 antibody and mouse anti-rat CD43 antibody (1:100) were added, and an overnight incubation at $4^{\circ} \mathrm{C}$ was performed. The processed samples were treated with fluorescein-tagged donkey anti-rabbit and donkey antimouse IgG antibodies (1:100) at room temperature for 1 h. Finally, DAPI was used to stain the nucleus. After being sealed with Antifade Mounting Medium, the cells were observed under a SP8 Lightning Confocal Microscope (LEICA).

\section{RT-qPCR Analyses}

Total RNA in the cells was extracted using TRNzol Universal reagent. The products were purified using pre-cooled $75 \%$ ethanol. After air drying, the residues were resolved in $20 \mu \mathrm{L}$ DEPC water. The purity and concentration of RNA were measured on a UV spectrophotometer (BioPhotometer, Eppendorf Scientific, Hamburg, Germany). The RNA was subsequently reverse transcribed to cDNA with a synthesis kit. Universal qPCR Master Mix kit was used in the quantitative PCR procedure on a 7500 Real-Time PCR Detection system (Applied Biosystems, Life Technologies) by taking the synthesized cDNA as templates, and relative mRNA expression was calculated based on $2^{-\Delta \Delta} \mathrm{CT}$ strategy with $\beta$ actin as the internal reference. Sequences of the oligonucleotide primers used are listed in Supplementary S2.

\section{Western Blot Experiments}

The cells were lysed in RIPA lysis buffer supplemented with phosphatase inhibitors and phenylmethanesulfonyl fluoride on ice. After a high-speed centrifugation $\left(12,000 \mathrm{rpm}\right.$ at $\left.4^{\circ} \mathrm{C}\right)$, the supernatant was collected, and protein contents were quantified using BCA kits. The samples were diluted with SDS-PAGE sample loading buffer, denatured in boiling water for $5 \mathrm{~min}$, and divided into aliquots for storage. Samples containing equal amounts of protein $(20 \mu \mathrm{g})$ were subjected to SDS-PAGE separation. The separated proteins were transferred onto polyvinylidene difluoride membranes. Thereafter, the membranes were treated with 5\% skim milk (at room temperature for $1 \mathrm{~h}$ ), anti-rat primary antibodies (at $4{ }^{\circ} \mathrm{C}$ overnight, 1:1000) and appropriate secondary antibodies (at room temperature for $2 \mathrm{~h}, 1: 5000$ ) in turns. The proteins were visualized using chemiluminescent reagent on an Amersham Imager 600 ECL Imaging System (GE). 


\section{Statistical Analysis}

The data were presented as mean \pm standard deviation. Results of the WB assays were semi-qualified with the aid of Image J (version 1.52a, NIH, Bethesda, MD, USA). Statistical differences among groups were analyzed using GraphPad Prism 8.0 (GraphPad Software, Cary, NC, USA) using one-way analysis of variance coupled with Tukey post hoc test.

\section{Results}

\section{QLY Treatment Affected Both T Cells and Monocytes in CIA Rats}

QLY treatment for $37 \mathrm{~d}$ effectively attenuated arthritic manifestation in CIA rats. Although inflammatory infiltration in subcutaneous tissues can still be noticed in QLYtreated CIA rats, cartilage erosion and rheumatoid nodule were not obvious (Figure 1A). As shown in Figure 1B, abnormally increased IL-17 $\alpha$ expression in the thymus of CIA rats was abrogated by QLY treatment. These findings were in line with the results of the flow cytometry analysis. Although $\mathrm{CD}^{+} \mathrm{CD}^{+} \mathrm{T}$ cells population was barely affected, compared with normal healthy controls, $\mathrm{CD}^{+} \mathrm{CD}^{+}{ }^{\mathrm{IL}}-17 \alpha^{+}$Th17 cell distribution was significantly increased in CIA rats $(2.3 \%$ vs $10.3 \%)$. QLY treatments significantly reduced both $\mathrm{CD}^{+} \mathrm{CD}^{+}$and $\mathrm{CD}^{+}{ }^{\mathrm{CD}} 4^{+} \mathrm{IL}-17 \alpha^{+}$cell subsets (Figure $1 \mathrm{C}$ ). In the cytokine chip analysis, 43 cytokines were successfully determined, and their levels were included in Supplementary $\underline{\mathrm{S} 3}$. The results confirmed the suppressive effects of QLY on Th17 cells, which was evidenced by decreased IL-22 (Figure 1D). ${ }^{34}$ The declined stem cell factor (SCF) and Flt-3L indicated that QLY could impair lymphocytes development. ${ }^{35}$ Hence, we subsequently investigated lymphocytes maturation-related factors in the marrow by immunohistochemical approach. The results indicated that the protein expression of SCF, Flt-3L and c-kit were up-regulated in CIA rats, while these proteins especially SCF and Flt-3L were suppressed by QLY treatment (Figure 1E). Similarly, RT-qPCR analysis revealed that QLY at $30 \mu \mathrm{g} / \mathrm{mL}$ reduced the mRNA expression of SCF in the marrow cells cultured in vitro (Figure $1 \mathrm{~F}$ ).

Decrease in activin A revealed by cytokine chip assay suggested that QLY could inhibit inflammatory polarization of monocytes. ${ }^{36} \mathrm{CD} 86$ is up-regulated during inflammation. It provides costimulatory signals for $\mathrm{T}$ cell activation. ${ }^{37} \mathrm{CD} 86$ was thus taken as a molecular marker of inflammatory monocytes in the subsequent flow cytometry assay. As expected, expanded population of $\mathrm{CD}^{+} 6^{+} \mathrm{CD} 11 \mathrm{~b}^{+}$monocytes was observed in CIA rats, which was reduced by QLY treatment (Figure $1 \mathrm{G}$ ). The inhibitory effects of QLY on inflammatory monocytes were validated by ELISA tests. Continuous QLY treatment significantly decreased serological levels of IL-1 $\beta$ and TNF- $\alpha$ (two typical inflammatory monocytes-released cytokines) in CIA rats (Figure $1 \mathrm{H}$ ). Compared with healthy controls, the level of IL-1 $\beta$ were significantly increased by almost 10 folds in CIA rats, which were subsequently reduced under QLY treatment. Comparatively, only 1.7-fold increase in TNF- $\alpha$ was observed in AIA rats. After QLY treatment, it was reduced to basal line.

\section{QLY Inhibited Monocytes-Mediated Inflammation in Active CIA}

Pathological changes in RA at different stages are governed by different immune cells., ${ }^{2,3}$ Even though we have proven that QLY treatment eventually improved immune conditions in CIA rats, the underlying mechanism is largely unknown. Hence, investigation on dynamic immune changes was necessary. Different from T cells, monocytes are mainly implicated in RA-related inflammation, and its involvement in early RA is not obvious. ${ }^{3}$ Accordingly, glycolysis-fueled inflammatory polarization of monocytes is a hallmark of active RA. ${ }^{38}$ Monitoring blood lactic acid therefore would differentiate stages of RA/CIA. Guided by the well-known progress pattern of CIA, we collected blood samples at four different time points and investigated cytokine and metabolite profiles using the serum to clarify the effects of QLY on different immune cellsgoverned disease stages.

As shown in Figure 2A, arthritic manifestation became visible on day 14. Since then, arthritis scores progressed rapidly and reached the peak on day 28. Afterwards, clinical manifestations were spontaneously alleviated. Accordingly, sampling points $1,2 / 3$ and 4 can be defined as onset, active and inactive phases of AIA, respectively. The lymphocyte counts in QLY-treated rats were not decreased initially, but an opposite outcome was observed. After $28 \mathrm{~d}$ treatment, QLY reduced the population of lymphocytes (Figure 2B). It implies that the therapeutic effects of QLY on CIA during early stages should not attribute to impaired proliferation and development of T cells. ELISA analyses indicated that the levels of IL-6, GM-CSF and IL-17 $\alpha$ in CIA rats were constantly and 


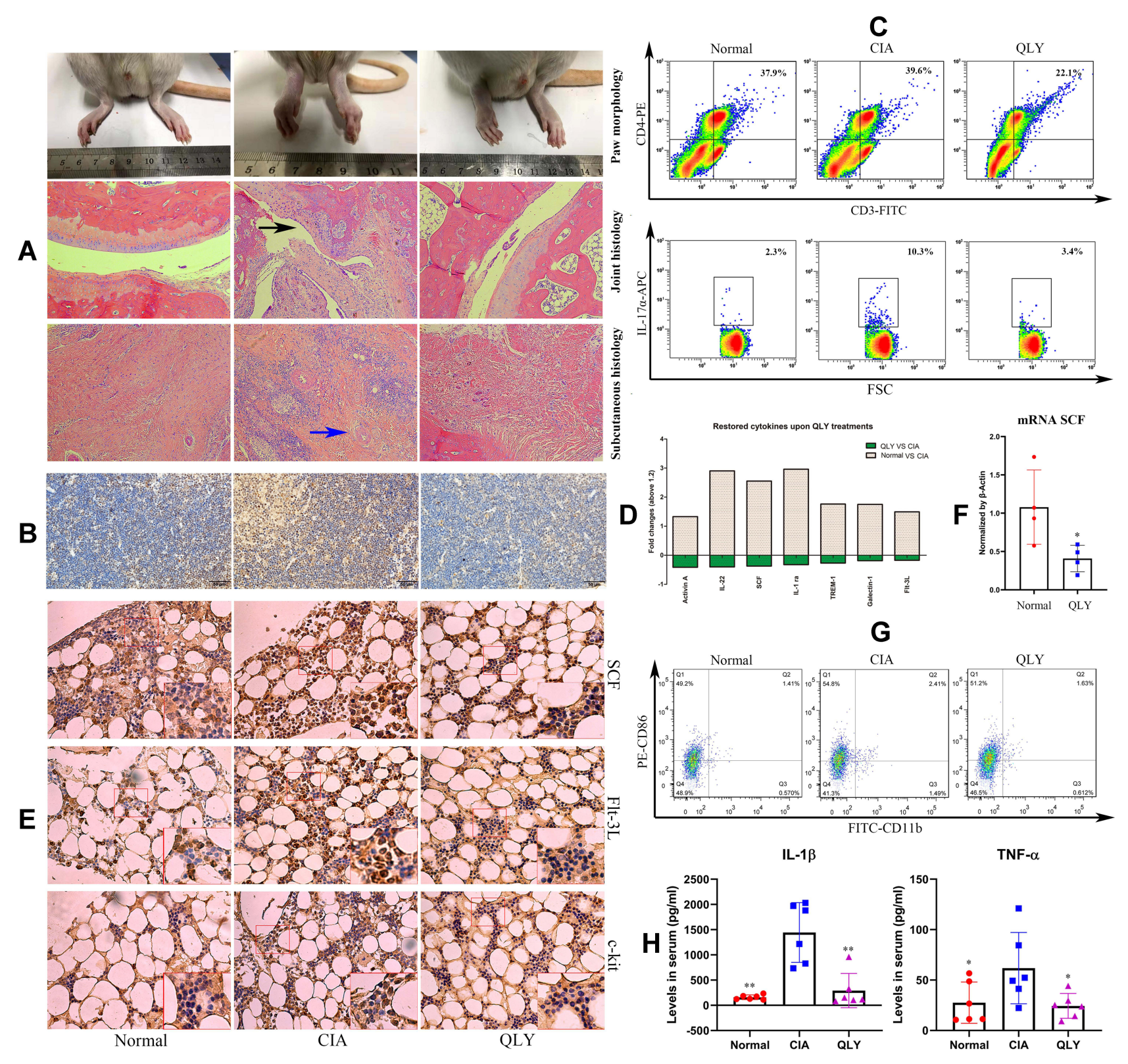

Figure I General effects of QLY treatment on CIA in rats. (A) Morphological observation of hind paws and historical examination of ankle joints/subcutaneous tissues, black arrow: cartilage erosion, blue arrow, rheumatoid nodules; (B) local expression of ThI7 $\alpha$ in the thymus assessed by immunohistochemical method; (C) distribution of $\mathrm{CD}^{+} \mathrm{CD} 4^{+} \mathrm{IL}-17 \alpha^{+}$Th 17 cells in peripheral blood evaluated by flow cytometry; (D) differentially expressed cytokines among different groups based on results from cytokine chip analysis; (E) protein expression of SCF, Flt-3L and c-kit in the marrow assessed by immunohistochemical method; (F) mRNA expression of SCF in marrow cells cultured in vitro evaluated by RT-qPCR; (G) distribution of inflammatory CD86 ${ }^{+}$CDI I b ${ }^{+}$monocytes in peripheral blood evaluated by flow cytometry; (H) levels of IL-I $\beta$ and TNF- $\alpha$ in the serum determined by ELISA. Statistical significance in image $(\mathbf{H}) *_{p}<0.05$ and $*^{*} p<0.01$ compared with $\mathrm{CIA}$ model rats; statistical significance in image $\mathrm{F}$, ${ }^{*}<0.05$ compared with normal untreated cells.

Abbreviations: QLY, Qing-Luo-Yin; CIA, collagen-induced arthritis.

significantly higher than those in the normal control. QLY treatment generally decreased all these $\mathrm{T}$ cells-released cytokines, but it exerted no effect on GM-CSF and IL$17 \alpha$ at stage 1 probably due to the increased lymphocytes counts (Figure 2C). Its effect on GM-CSF was weakened at stage 4 , while its inhibitory effects on IL-17 $\alpha$ and IL-6 production still persisted. GM-CSF is a potent inducer of inflammatory polarization of monocytes, which promotes the expression of HLA-DR and CD86, as well as increases the production of TNF- $\alpha$ and IL- $1 \beta .{ }^{39}$ These findings suggested that QLY treatment was especially effective in easing monocytes-mediated inflammation during active AIA (stage 2-3). The overall evidences also demonstrated that since the onset of AIA, T cell hyperactivation had occurred, which could subsequently activate monocytes by releasing cytokines like GM-CSF. 
A

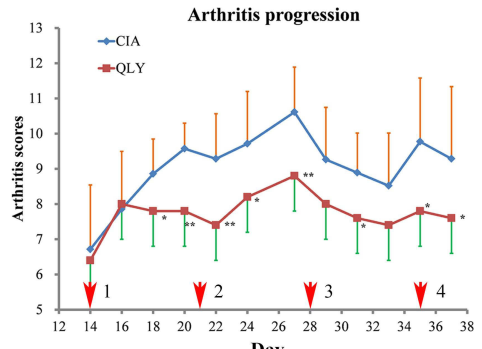

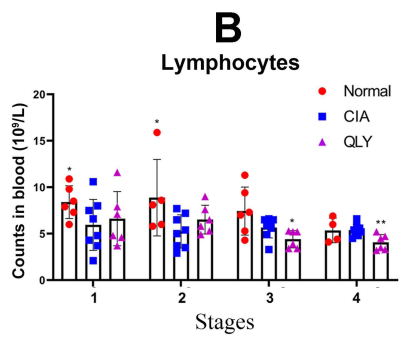

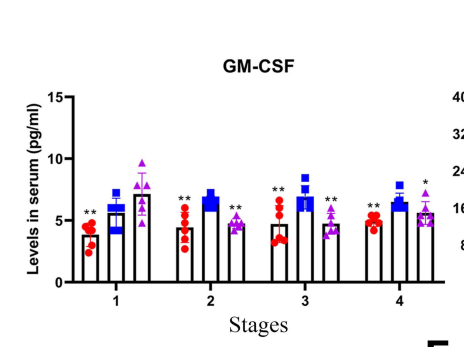

D

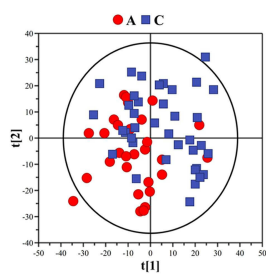

Normal VS CIA

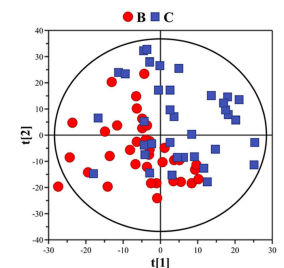

CIA VS QLY

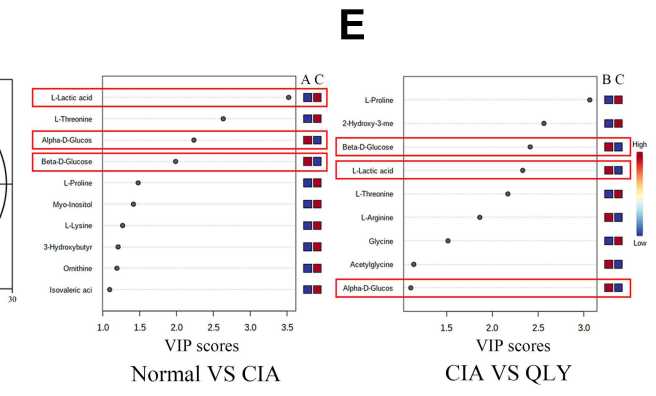

LL-6

C

$\mathbf{F}$
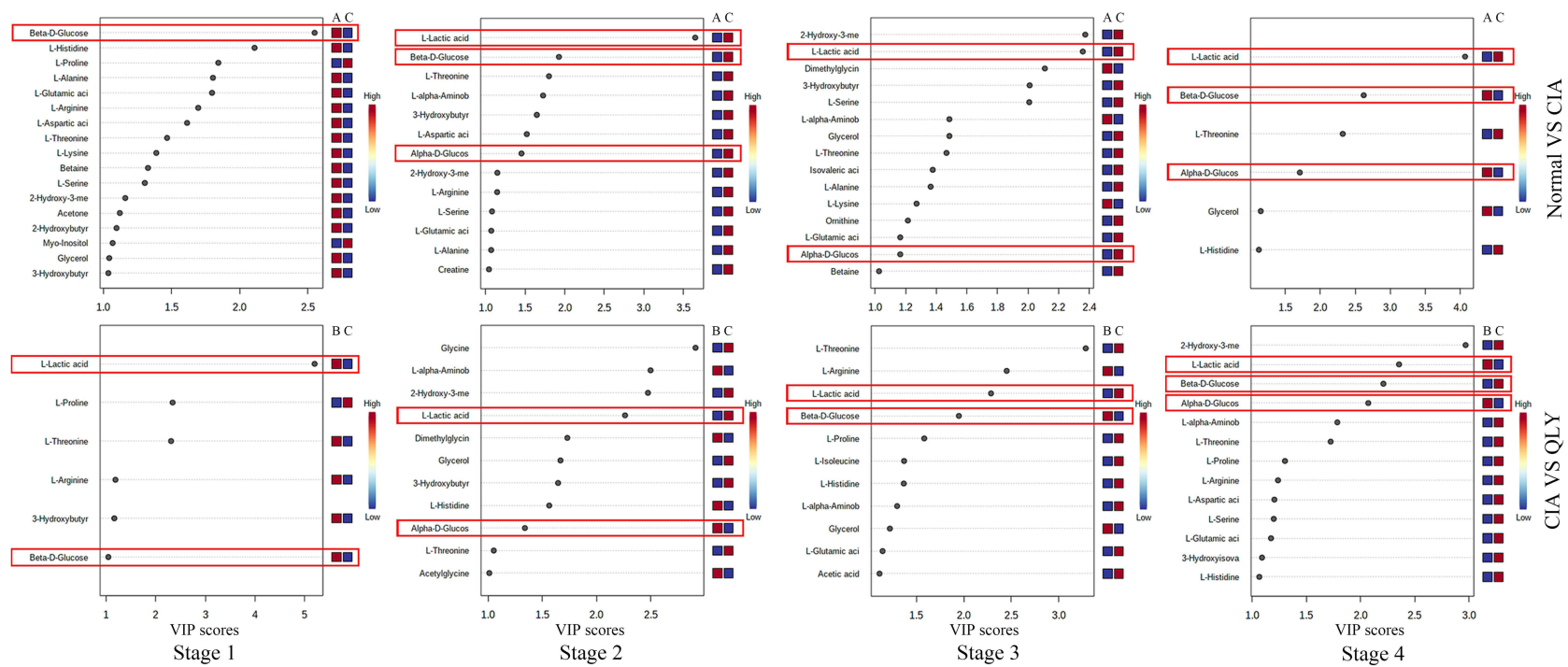

Figure 2 Dynamic changes of arthritic severity and metabolic profile in CIA rats (stage I: AIA onset, stages 2-3, active phase, stage 4, inactive phase). (A) Periodic changes in arthritis score throughout the observational period; (B) periodic changes in peripheral lymphocyte counts; (C) periodic changes in T cells-released inflammatory cytokines in peripheral blood assessed by ELISA; (D), general metabolic profile differences among different groups revealed by PLS-DA using 'H NMR data of the serum; (E), general discriminating metabolites among different groups revealed by PLS-DA using data from all the samples (ranked according to VIP values); (F), discriminating metabolites among groups identified by PLS-DA at different sampling time points. The symbols of A, B, and C in images (D-F) represent normal healthy, QLY treatment and CIA groups, respectively. Statistical significance: ${ }^{*} p<0.05$ and $*^{*} p<0.01$ compared with AIA models.

Abbreviations: QLY, Qing-Luo-Yin; CIA, collagen-induced arthritis; PLS-DA, partial least squares-discrimination analysis; VIP, variable importance in projection.

The representative ${ }^{1} \mathrm{H}$ NMR spectrums of the blood samples are shown in Supplementary S4. A total of 47 metabolites were identified, and the signal assignments are shown in Supplementary S5. The samples collected at different time points from identical groups were firstly assigned into one group. The results from PLS-DA revealed differed metabolic profiles among groups (Figure 2D). The top discriminating metabolite between normal and CIA rats was lactic acid. Meanwhile, significant decrease in the blood glucose level was observed in
CIA rats. QLY treatment greatly affected the amino acid composition in the serum of CIA rats. Particularly, proline was decreased by QLY, which supported the protective effects of QLY on joints, as proline is a characteristic component of CII. ${ }^{40}$ QLY reversed blood glucose decline. However, confusingly, it did not inhibit but further promoted the overall lactic acid production in CIA rats (Figure 2E). Afterwards, we exhibited the dynamic metabolic changes in Figure 2F. Since stage 2, compared with healthy controls, consistently higher levels of lactic acid 
were observed in CIA rats. It was accompanied with obvious blood glucose decline, demonstrating accelerated aerobic glycolysis. Because rheumatic $\mathrm{T}$ cells rely on pentose phosphate pathway (PPP) for energy supply, it further confirms that glycolysis-fueled inflammation in CIA rats is mainly driven by monocytes. ${ }^{38,41}$ QLY suppressed lactic acid production during stages $2-3$, but it exerted the opposite effects at stages 1 and 4 . The results partially explained the abnormal increase of lactic acid in QLY-treated CIA rats in total samples (Figure 2E). More importantly, it indicated that QLY treatment mainly inhibited inflammatory monocytes during active CIA.

\section{QLY Inhibited Inflammatory Monocytes in Active AIA}

The findings stated above showed that QLY treatment improved the immune environment of rheumatic subjects by simultaneously targeting $\mathrm{T}$ cells and monocytes, but its effects on acute inflammation are largely mediated by monocytes. To confirm this conclusion, we subsequently performed experiments on AIA model, which is mainly mediated by inflammatory monocytes. ${ }^{28}$ As the therapeutic effects of QLY on AIA have been reported, herein we mainly emphasized immune changes by analyzing blood collected on day 18 (the peak of inflammation). ${ }^{42}$ Flow cytometry analysis demonstrated that similar to its effects on CIA rats, QLY reduced $\mathrm{CD} 3^{+} \mathrm{CD} 4^{+} \mathrm{IL}-17 \alpha^{+} \mathrm{T}$ cells population in AIA rats (Figure 3A). Inflammatory $\mathrm{CD} 8{ }^{+} \mathrm{CD} 11 \mathrm{~b}^{+}$monocytes were significantly increased in AIA rats, which were effectively suppressed by QLY treatment (Figure 3B). CD172a and CD43 are two commonly used phenotype markers for rat monocyte classification. $^{43}$ It was obvious that the total CD $172 \mathrm{a}^{+} \mathrm{CD} 43^{+}$monocytes were increased in AIA rats, and their counts were slightly reduced by QLY. Because of limited population, they can hardly be further divided into CD172 $\mathrm{a}^{+} \mathrm{CD} 43^{\mathrm{dim}}$ and $\mathrm{CD} 172 \mathrm{a}^{+} \mathrm{CD} 43^{\text {bright }}$ subsets. Functional phenotypes of monocytes during active AIA were further investigated. PCR analysis using blood sampled on day 18 revealed significantly increased mRNA expression of iNOS, IL-1 $\beta$ and MCP-1 in AIA WBCs. Meanwhile, the anti-inflammatory molecular markers IL-10, Arg-1 and SIRT1 were barely affected (Figure 3C). It confirmed that monocytes during active AIA were predominantly inflammatory. QLY treatment induced profound decrease in these inflammatory indicators. At the same time, it promoted the expression of IL-
10, Arg-1 and SIRT1. On day 28 (ten days later), by the spontaneous remission of inflammation, AIA progressed to inactive stages. The increase in mRNA expression of iNOS, IL-1 $\beta$ and MCP-1 was not very significant, but notable deficiency in the expression of IL-10 and Arg-1 occurred. At this time point, although anti-inflammatory properties of QLY can still be noticed, its effects on iNOS, IL-1 $\beta$ and MCP-1 were weakened (Figure 3C). These evidences demonstrated that inactive AIA could be caused by the deficiency in anti-inflammatory monocytes, and QLY mainly inhibited inflammatory monocytes during the active stages. As illustrated in Figure 3D, increased inflammatory CD43 ${ }^{\mathrm{dim}}$ monocytes were identified in AIA blood collected on day 18 , and the size was bigger than the cells derived from either normal or QLY-treated CIA rats. It was observed that the expression of SIRT1, a negative regulator of inflammatory polarization was low in AIA monocytes, while QLY treatment significantly promoted it. $^{44}$ Meanwhile, we only observed non-inflammatory CD43 ${ }^{\text {bright }}$ monocytes in samples from QLY-treated CIA rats. ${ }^{43}$ It suggested that QLY therapy favored the development of these cells.

\section{QLY Disrupted Inflammatory Interplay Between Lymphocytes and Monocytes}

As AIA is mainly driven by inflammatory monocytes, the decrease in Th17 cells under QLY treatment could be largely mediated by monocytes. To test this speculation, we cotreated normal splenocytes with different rats-derived blood monocytes and analyzed Th17 cell distribution. As expected, AIA monocytes facilitated Th17 differentiation in the cocultured splenocytes, while QLY greatly impaired their potentials (Figure 4A). The changes in $\mathrm{CD}^{+} \mathrm{CD}^{+}$cell frequency further proved that the negative effects of QLY on lymphocyte development can only be achieved under repeated/intense stimulus. Compared with normal control cells, splenocytes co-cultured with AIA monocytes released higher levels of IL-6 and IL-1 $\beta$, which was inhibited by QLY treatment (Figure 4B).

Meanwhile, the suppression on inflammatory monocytes under QLY treatment could also be mediated by the effects on lymphocytes too, considering that RA is a T cells-driven disease. Subsequently, we co-cultured normal monocytes with AIA lymphocytes in the presence of different rat serum. PCR analysis showed that AIA lymphocytes induced obvious increases in the expression of iNOS and IL-1 $\beta$ in normal monocytes, while IL-10 and SIRT1 expression was 

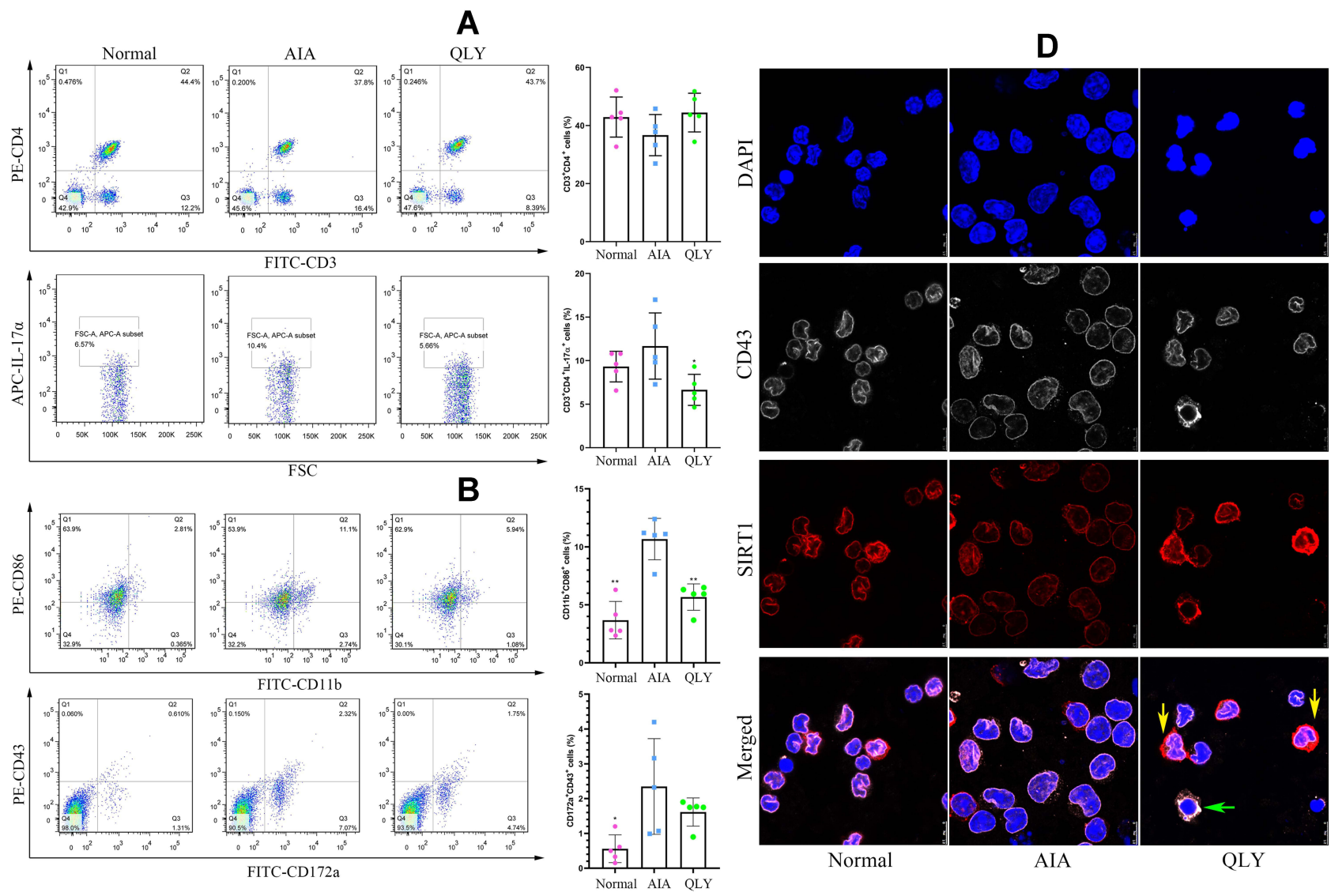

C
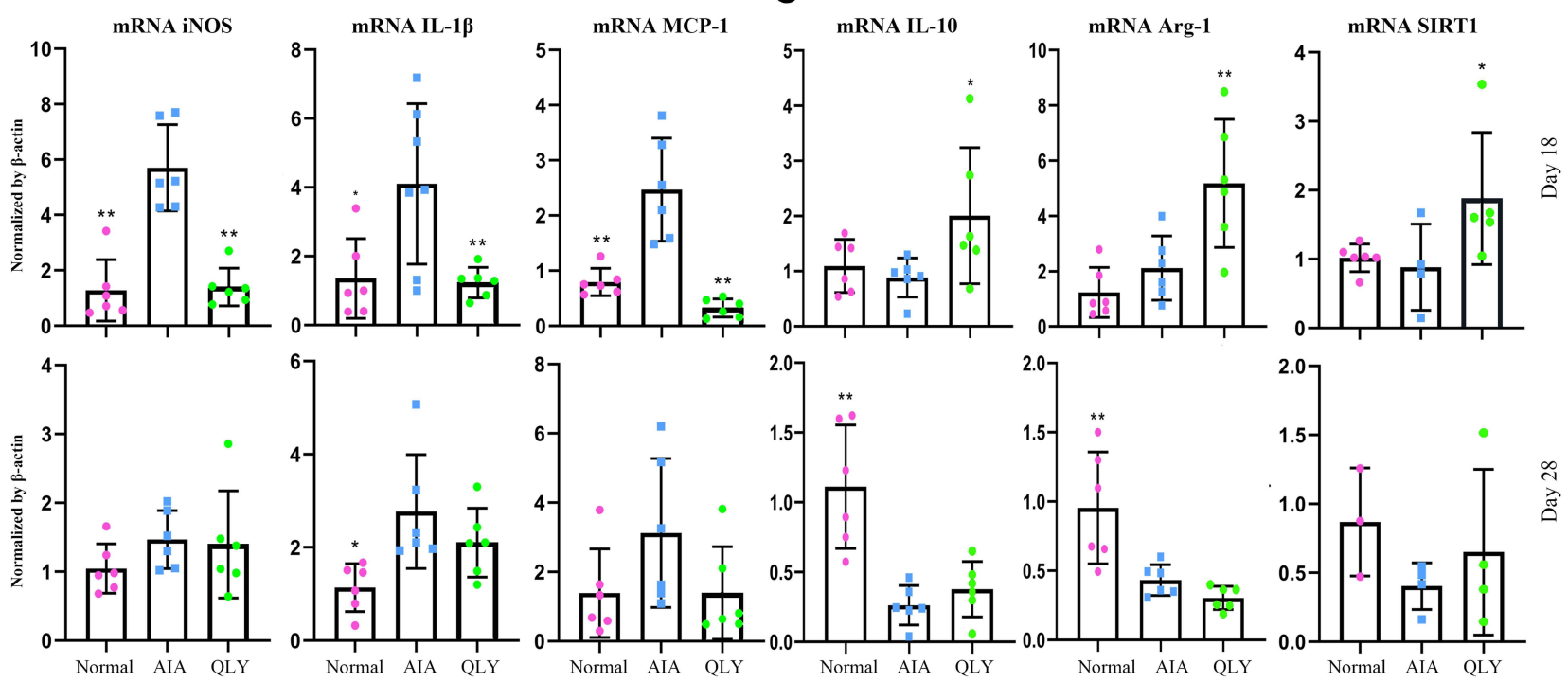

Figure 3 Impact of QLY treatment on immune milieu in AIA rats. (A) Flow cytometry analysis of CD3 ${ }^{+} \mathrm{CD} 4^{+} \mathrm{IL}-17 \alpha^{+}$ThI7 cells in peripheral blood sampled on day I8; (B) flow cytometry analysis of inflammatory CD $43^{+} \mathrm{CD} 86^{+}$monocytes in peripheral blood sampled on day I8; (C) mRNA expression of iNOS, IL-I $\beta$, MCP-I, IL-I0, Arg-I, SIRT I in peripheral WBCs collected on day 18 and 28 assessed by RT-qPCR; (D) immunofluorescence method-based observation of co-localization of molecule CD43 and SIRTI in peripheral monocytes sampled on day 18, yellow arrow: high expression of SIRTI, green arrow: high expression of CD43. Statistical significance: *p < 0.05 and $*^{*} p<0.01$ compared with AIA model rats.

Abbreviations: QLY, Qing-Luo-Yin; AIA, adjuvant-induced arthritis.

inhibited. QLY-containing serum reshaped AIA lymphocytes-stimulated monocytes towards the anti-inflammatory phenotype (Figure 4C). Accordingly, AIA lymphocytes- induced increase in IL- 6 and IL- $1 \beta$ secretion in monocytes was also inhibited by QLY-containing serum (Figure 4D). We subsequently investigated two key regulators involved in 


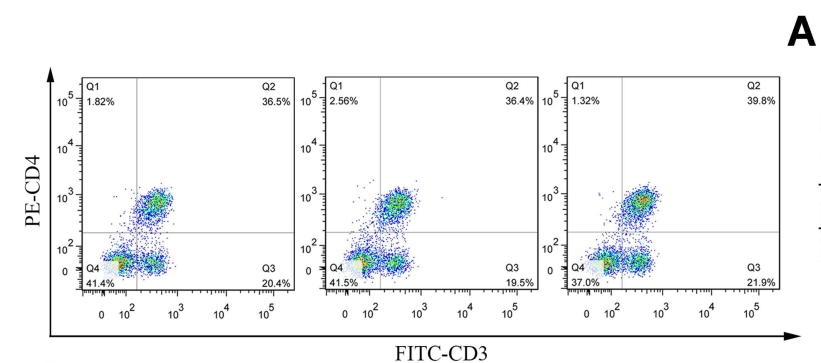

A
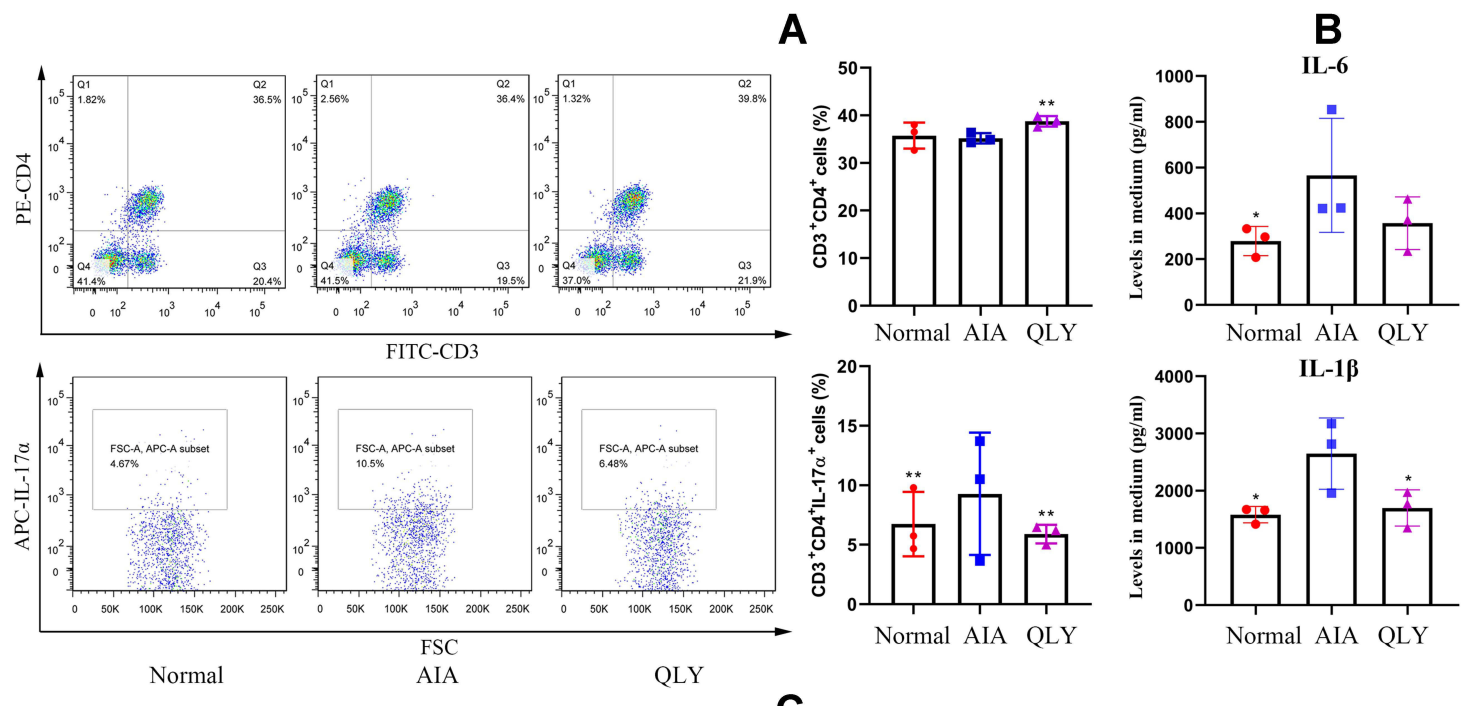

C

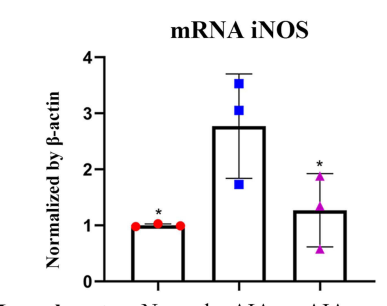

Lymphocytes Normal AIA AIA
Serum Normal Normal QLY
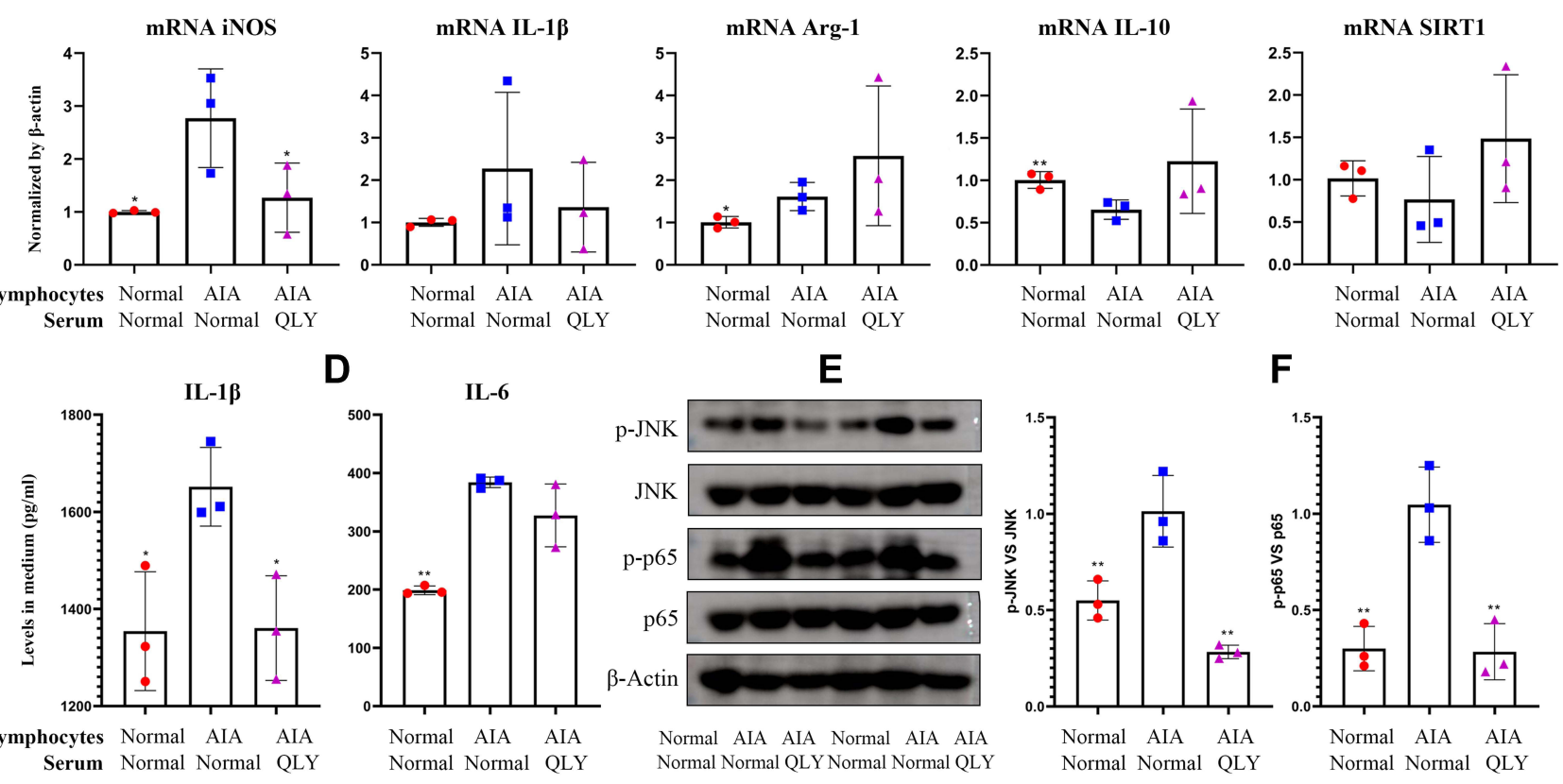

D

E
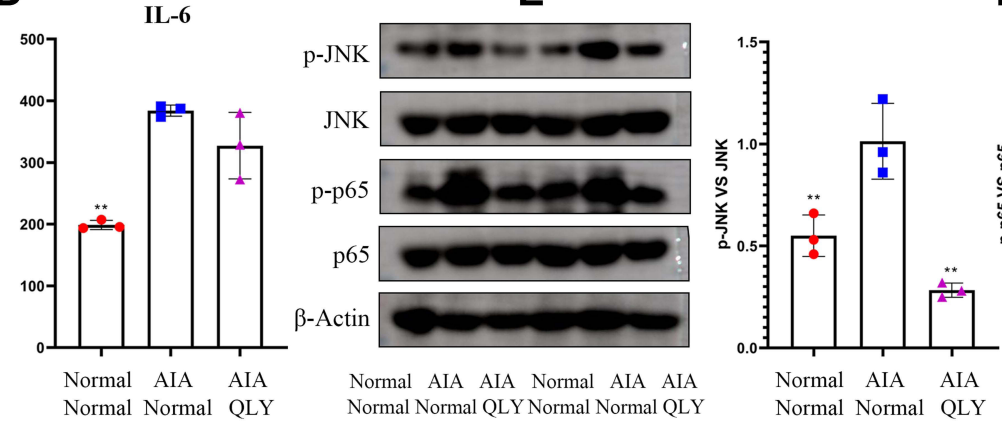

$\mathbf{F}$

Figure 4 Interplay between lymphocytes and monocytes during QLY treatments in AIA. (A) Flow cytometry analysis of CD $3^{+} \mathrm{CD} 4^{+} \mathrm{IL}-\mathrm{I7} \alpha^{+} \mathrm{ThI} 7$ cells in normal splenocytes co-cultured from monocytes from different rats; (B) levels of IL- 6 and IL-I $\beta$ released by splenocytes co-cultured from monocytes derived from different rats, determined by ELISA; (C) mRNA expression of iNOS, IL-I $\beta$, IL-I0, Arg-I, SIRTI in normal monocytes co-cultured with either normal or AIA lymphocytes in the presence of different rat serums assessed using RT-qPCR; (D) levels of IL-6 and IL-I $\beta$ released by normal monocytes co-cultured with either normal or AIA lymphocytes in the presence of different rat serums determined by ELISA; (E) Western blot analysis of p65, p-p65, JNK, p-JNK expression in normal monocytes co-cultured with either normal or AIA lymphocytes in the presence of different rat serums; $(\mathbf{F})$ quantification of assay E. Statistical significance: $*_{p}<0.05$ and **p $<0.01$ compared with the cells co-cultured with immune cells derived from AIA rats.

Abbreviations: QLY, Qing-Luo-Yin; AIA, adjuvant-induced arthritis.

monocyte polarization using WB analysis (Figure 4E). AIA lymphocytes facilitated the phosphorylation of JNK and p65 in the co-cultured monocytes, which was abrogated by QLYcontaining serum (Figure 4F). These results confirmed that the negative effects of QLY on inflammatory monocytes are at least partially mediated by lymphocytes.

\section{Alkaloid Derivatives are the Main Anti-Rheumatic Components Within QLY}

Using the previously reported bioactive compounds in QLY and their therapeutic targets, we performed pathway enrichment. KEGG annotation revealed the potential effects of 
QLY on RA pathway (Figure 5A). GO-based analysis demonstrated that the effects of QLY on lipopolysaccharide (LPS) responses significantly contributed to its medicinal properties (Figure 5B). These results were supported by the in vivo experiments stated above, because $\mathrm{T}$ cells-related genes were made up of the majority of RA pathway, and LPS is a key inducer of inflammatory polarization of monocytes. Afterwards, we displayed the interactions between compounds and corresponding genes within RA and LPS pathways separately (Figure 5C and D). ID numbers for the compounds are shown in Supplementary S7. The importance of each compound was ranked by their relevance to the signaling network, which was reflected by the varied size of plot in diagram. In the LC-MS experiments, we analyzed chemical compositions of QLY decoction and QLYcontaining serum. The top 25 components with high abundances from each analysis are listed in Figure 5E. We were especially interested in the abundant compounds showing potent effects on T cells and monocytes-related genes. Based on the convergence of their distribution in the decoction and serum and the relevance to above pathways, we identified three components, namely SIN, MT and SCA. Quantitative
A
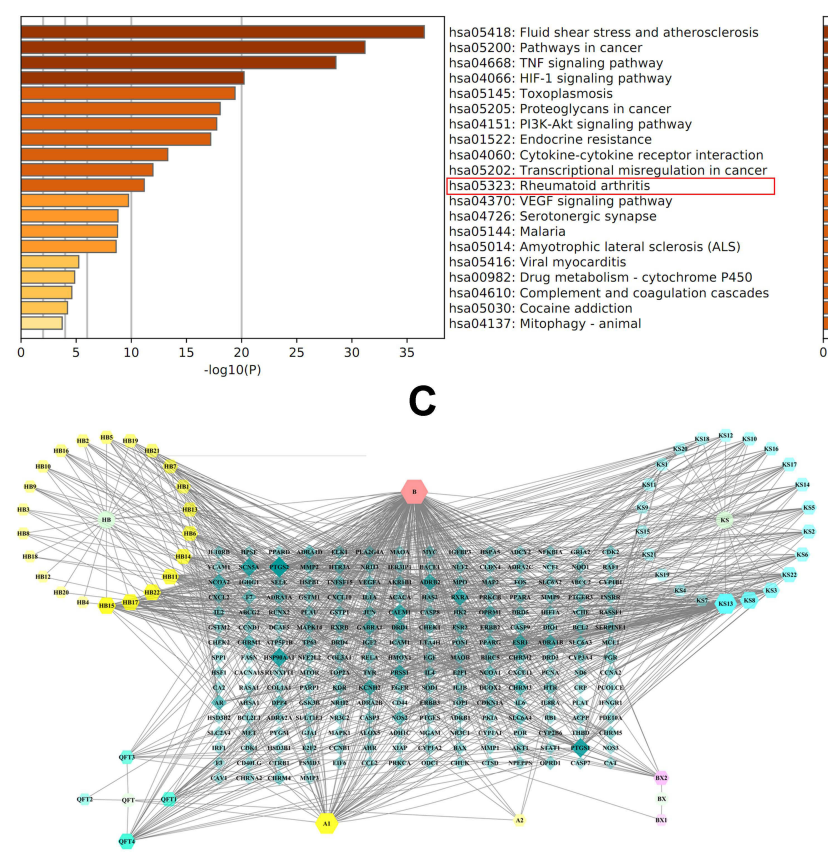

B
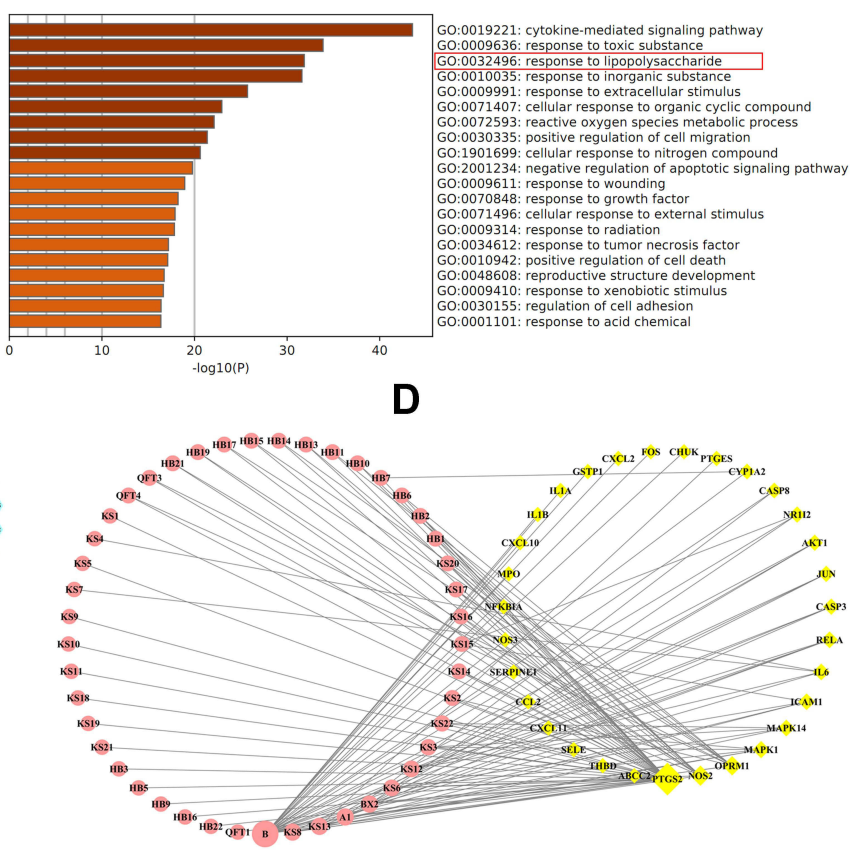

E

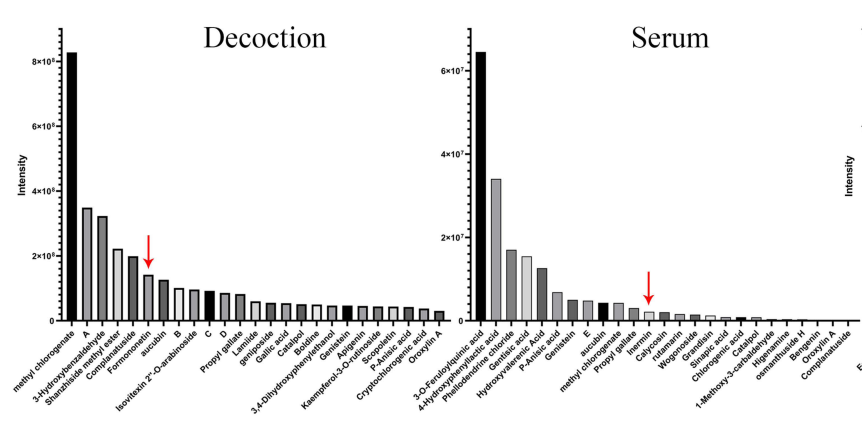

Negative mode

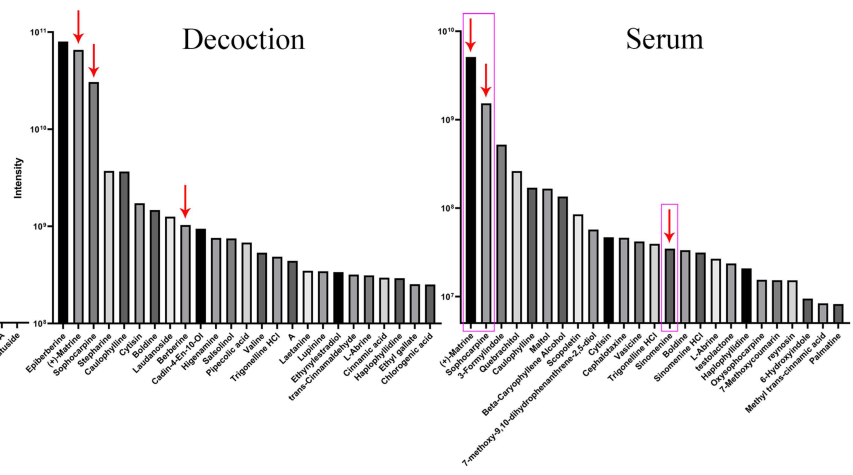

Positive mode

Figure 5 Identification of potential anti-rheumatic components from QLY decoction. (A) KEGG pathway enrichment analysis based on reported target genes of compounds within QLY; (B) GO pathway enrichment analysis based on reported target genes of compounds in QLY; (C) interactions between bioactive compounds and corresponding target genes from RA pathway; (D) interaction between bioactive compounds and corresponding target genes from LPS response pathway; (E) top 25 abundant chemical components detected in QLY decoction and QLY-containing rat serum, ranked by their relative intensities in total ion chromatograms of LC-MS analysis, red arrow: RA/LPS pathway-related compounds with relative high abundance in both QLY decoction and QLY-containing serum.

Abbreviations: QLY, Qing-Luo-Yin; AIA, adjuvant-induced arthritis, RA, rheumatoid arthritis; KEGG, Kyoto Encyclopedia of Genes and Genomes; GO, Gene Ontology; LPS, lipopolysaccharide. 
analysis indicated that their contents in QLY-containing serum were $67.2,18.3$ and $92.5 \mathrm{ng} / \mathrm{mL}$, respectively (Supplementary S8). These alkaloids can target $\mathrm{T}$ cells and monocytes (Figure 5C and D) and are easily absorbed after oral administration (Figure 5E). These findings were solidly supported by relevant literatures. ${ }^{24-26}$

\section{QLY-Derived Alkaloids Preferentially Inhibited Inflammatory T Cells}

We firstly treated blood-derived AIA lymphocytes with the identified compounds using concentrations detected in QLYcontaining serum (serum concentrations). Compared with normal controls, AIA lymphocytes obviously secreted higher IFN- $\gamma$ (223.5 vs $20.2 \mathrm{pg} / \mathrm{mL})$. Treatment with these compounds for $12 \mathrm{~h}$ achieved substantial reduction in IFN- $\gamma$ secretion. Similar efficacies were observed under SIN (80 $\mathrm{ng} / \mathrm{mL})$, MT (100 ng/mL) and combination treatments, while SCA $(20 \mathrm{ng} / \mathrm{mL})$ stimulus achieved a weaker effect (Figure 6A). Due to low basal levels, differences in IL-17 $\alpha$ among the groups were not obvious, and results from this ELISA assay were not very convincing. Similarly, limited amounts of Th17 cells in the lymphocytes did not allow us to perform flow cytometry analysis. Alternatively, we investigated p-STAT3 (a key regulator of Th17 differentiation) to characterize changes of this subset (Figure 6B). ${ }^{45}$ All treatments effectively suppressed the abnormally increased phosphorylation of p-STAT3 in AIA lymphocytes (Figure 6C). It convinced us that QLY inhibited the development of inflammatory $\mathrm{T}$ cells like Th1/17 cells in AIA rats. The effects on $\mathrm{T}$ cells were further supported by PCR analysis. Increase in the mRNA expression IFN- $\gamma$ and IL-17 $\alpha$ in AIA lymphocytes was abrogated by all the compounds (Figure 6D). More profound changes in the mRNA expression of IFN- $\gamma$ indicated that Th1 rather than Th17 cells were the dominant inflammatory T cells in AIA rats. Although the combined use of these compounds did not bring any tangible benefits concerning their effects on inflammatory $\mathrm{T}$ cells, it further restored the declined expression of anti-inflammatory indicators SIRT1 and IL-4.

Next, we investigated the effects of these alkaloids on monocytes. To our disappointment, PCR analysis showed that none of these compounds at serum concentrations affected inflammatory AIA monocytes (Figure 7A). However, the situation was changed when treatment concentrations were increased by 20 -fold. All the treatments inhibited the mRNA expression of iNOS and IL-1 $\beta$ more or less. Besides, the mRNA expression of SIRT1, Arg-1 and IL-10 in AIA monocytes were significantly promoted by SIN, MT and SCA, and their combination even further amplified this regulation (Figure 7A). Eventually, it led to profound decrease in IL-1 $\beta$ production (Figure 7B). But all these treatments did not suppress the increased IL-6 production. We subsequently investigated two regulators of inflammatory polarization by WB (Figure 7C). Although all the compounds inhibited the phosphorylation of p65 and JNK to certain extent, their synergistic effects showed

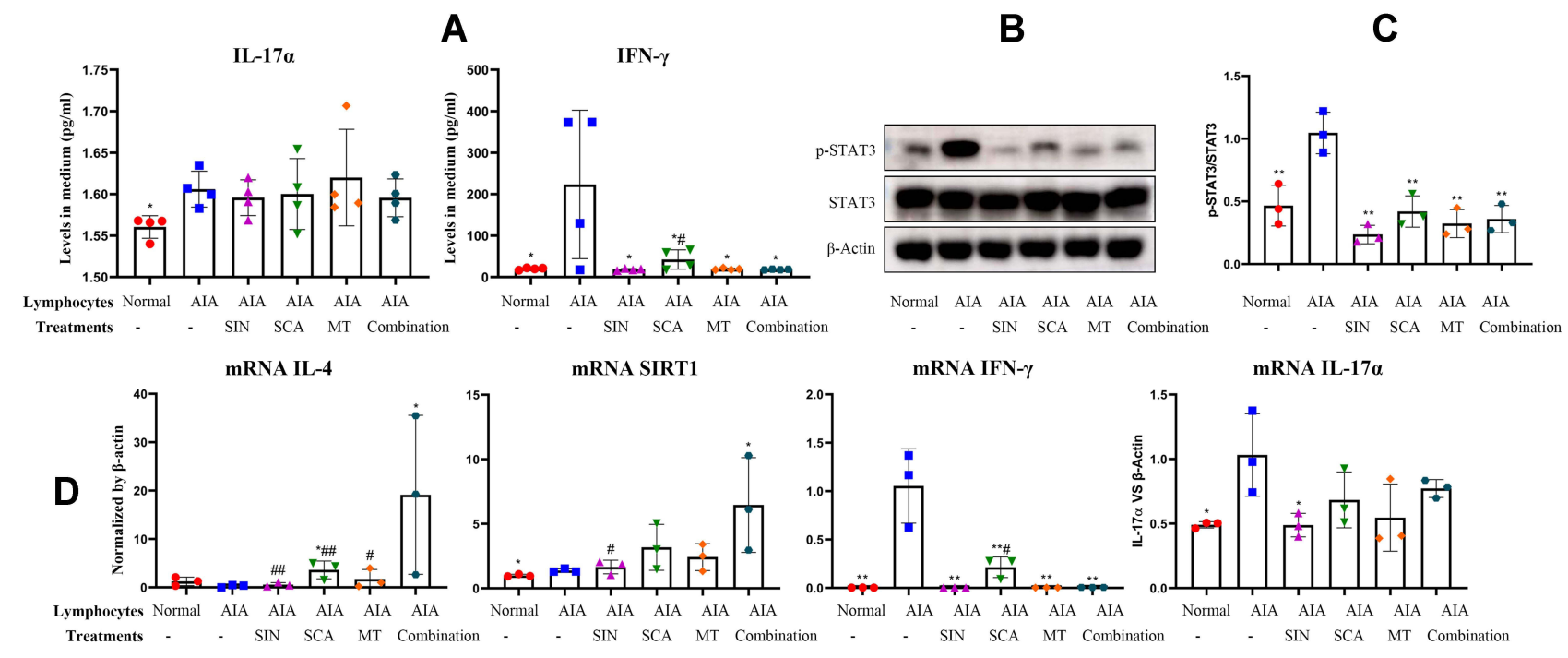

Figure 6 Effects of QLY-related anti-rheumatic compounds on AIA lymphocytes in vitro. (A) Levels of IFN- $\gamma$ and IL-I7 $\alpha$ in the medium released by compounds-treated AIA lymphocytes, determined by ELISA; (B) expression of protein (p)-STAT3 in compounds-treated AIA lymphocytes, assessed by Western blot analysis; (C) quantification of assay B; (D) mRNA expression of IL-4, SIRTI, IFN- $\gamma$ and IL-I $7 \alpha$ in compounds-treated AIA lymphocytes, evaluated by RT-qPCR. Statistical significance: *p < 0.05 and **p < 0.01 compared with untreated AIA lymphocytes; ${ }^{*}<<0.05$ and ${ }^{\#} \mathrm{P}<0.01$ compared with AIA lymphocytes receiving combination stimulus (SIN + SCA + MT). Abbreviations: QLY, Qing-Luo-Yin; AIA, adjuvant-induced arthritis; SIN, sinomenine; SCA, sophocarpine; MT, matrine. 

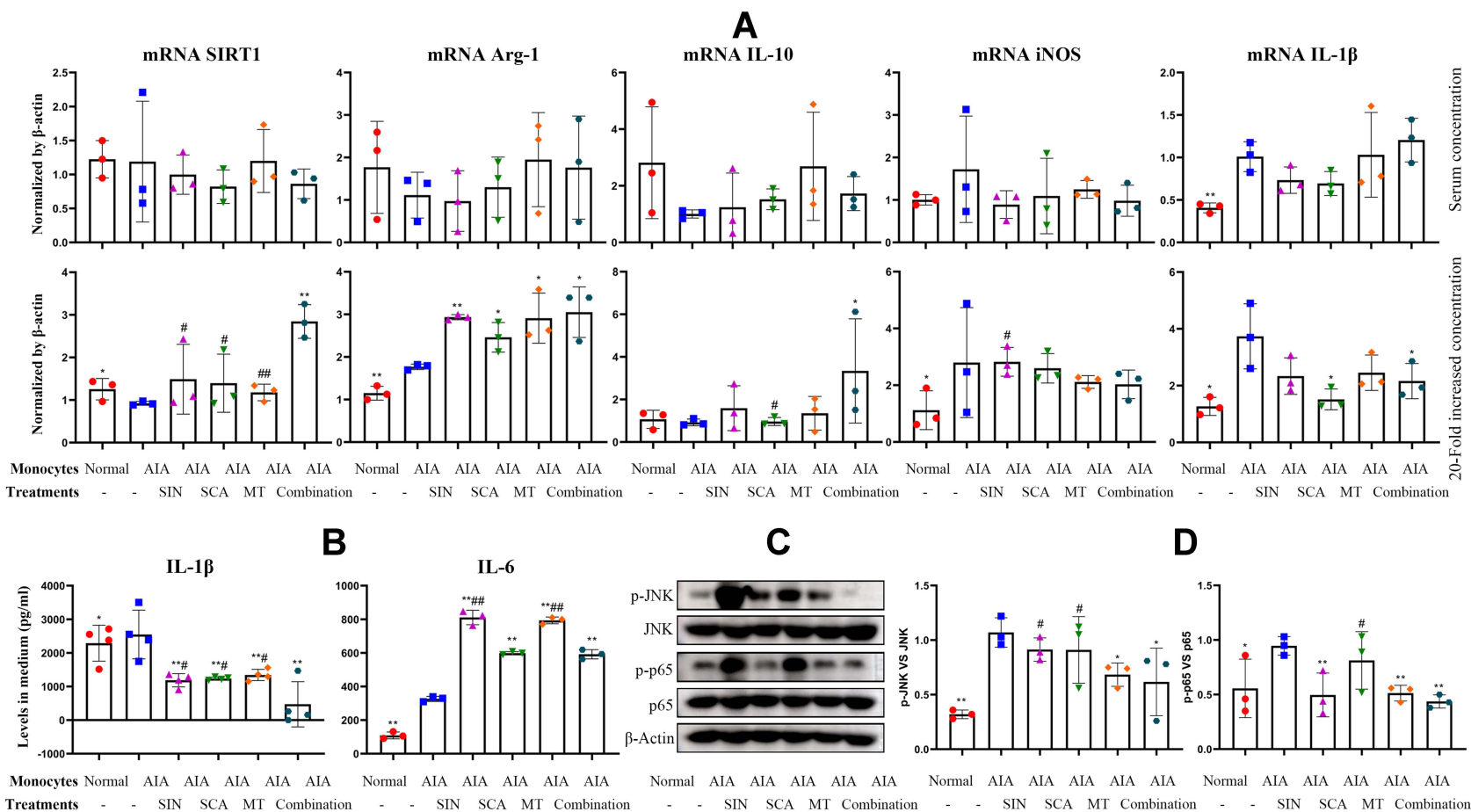

Figure 7 Effects of QLY-related anti-rheumatic compounds on AIA monocytes in vitro. (A) mRNA expression of SIRTI, Arg-I, IL-I0, iNOS and IL-I $\beta$ in compounds-treated AIA monocytes using concentrations detected in QLY-containing serum (serum concentration) or concentrations increased by 20 -folds (20-fold increased concentration); (B) levels of IL-I $\beta$ and IL-6 in medium released by compounds-treated AIA monocytes using 20 -fold increased concentration, determined by ELISA; (C) Western blot analysis of p65, p-p65, JNK, p-JNK protein expression in compound-treated AIA monocytes using 20-fold increased concentration; (D) quantification of assay C. Statistical significance: ${ }^{*} \mathrm{p}<0.05$ and ${ }^{* *} \mathrm{p}<0.01$ compared with untreated AIA monocytes; ${ }^{\#} \mathrm{p}<0.05$ and ${ }^{\# \#} \mathrm{p}<0.01$ compared with AIA monocytes receiving combination stimulus $(\mathrm{SIN}+\mathrm{SCA}+\mathrm{MT})$

Abbreviations: QLY, Qing-Luo-Yin; AIA, adjuvant-induced arthritis; SIN, sinomenine; SCA, sophocarpine; MT, matrine.

more profound decrease in p-p65 and p-JNK expression (Figure 7D). Generally, the direct effects of the chemical stimuli on AIA monocytes were weaker than those observed in T cells.

\section{Discussion}

Arthritic manifestation of RA is mainly driven by synovitis, which provokes persistent inflammation and progressive tissue degradation in joints. ${ }^{1-3}$ Undoubtedly, the breakdown of immune homeostasis is the leading cause of synovitis, while the role of angiogenesis is similarly important. Evidences showed that QLY treatment can reduce the production of inflammatory mediators, such as C-reactive protein, rheumatoid factors, TNF- $\alpha$, IL-17 and IL-6. ${ }^{23,46}$ Increase in angiogenesis indicators like vascular endothelial growth factor can also be suppressed by this regimen. ${ }^{46,47}$ It has been proven that QLY-induced disruption in Ang/Tie2 pathway eventually impaired proliferation, migration and tube formation capability of vascular endothelial cells. ${ }^{47}$ These findings basically validated its therapeutic effects on RA, while key upstream immune events accounting for these beneficial changes were not been identified. Our recent work suggested that metabolic reprogramming could be important for RA-related angiogenesis, and the accelerated aerobic glycolysis is a driving force for this abnormality. ${ }^{48}$ It encourages us to explore the therapeutic mechanism of QLY from the angle of energy metabolism.

As a cold-natured formula, the therapeutic actions of QLY were distinctively different from those with hot properties. ${ }^{47}$ To better clarify its cold-natured properties, we previously investigated the metabolic changes in QLYtreated CIA rat. ${ }^{49}$ The most impressive metabolic alterations were identified as down-regulated nucleic acid synthesis and PPP. The depletion of pyrimidine and purine derivatives would ease the hyperactivated immune status in RA patients, as they are indispensable for lymphocytes replicating. ${ }^{50}$ More importantly, QLY-impaired PPP will impede the proliferation and activation of $\mathrm{T}$ cells. ${ }^{41}$ These clues-revealed suppressive effects of QLY on pathogenic lymphocytes were evidenced by the decrease in the expression of SCF, Flt-3L, c-kit (Figure 1D and E), blood lymphocyte counts (Figure 2B) and Th17 cell frequency (Figures 1C and 3A) in this study. Eventually, QLY 
treatment led to significant decrease in $\mathrm{T}$ cells-released cytokines (Figure 2C).

The current ${ }^{1} \mathrm{H}$ NMR-based metabolomics study further identified monocytes as an important target of QLY. Due to energy generation speed merits, inflammatory monocytes utilize glucose mainly through aerobic glycolysis. ${ }^{38,51}$ During active CIA, accumulated lactic acid was significantly reduced by QLY therapy (Figure 2F), demonstrating its potent effects on inflammatory metabolic reprogramming. Its effects on monocytesmediated inflammation were subsequently validated in active AIA (Figure 3C). Theoretically, the inhibition on glycolysis would prevent monocytes acquiring inflammatory phenotype, because inflammatory events require stable energy supply. ${ }^{41,51}$ The current study suggests that QLY may inhibit the glycolysis of monocytes by upregulating SIRT1 (Figures 3C and 4C). SIRT1 serves as a switch of glycolysis and oxidative phosphorylation. It is directly involved in transcriptional regulation of glycolytic regulators. ${ }^{41}$ Besides, it can inhibit NF- $\mathrm{BB}$ and AP-1 pathways and indirectly affecting metabolic profile. ${ }^{41,51}$ In short, SIRT1 activation is unfavorable for inflammatory polarization. Interestingly, we found that QLY treatment promoted SIRT1 expression in all monocytes including CD43 ${ }^{\text {dim }}$ monocytes, which are usually categorized as inflammatory subset (Figure 3D). Because SIRT1 had profound impact on the general functional phenotype of monocytes (Figure 3C), this fact implies that QLY-caused changes on SIRT1 rather than surface molecules decided the fate of monocytes in rheumatic subjects.

As for how QLY promoted SIRT1 expression in monocytes, there are many possibilities. As shown in Figure 7A, intense stimulus with the compounds from QLY can directly increase the mRNA expression of SIRT1. However, we have to admit this effect was weak and cannot be achieved using serum concentrations. Comparatively, lymphocytes-mediated theory is more convincing. As well known, inflammatory polarization of monocytes in RA is mainly mediated by lymphocytes. ${ }^{3}$ Pathogenic $\mathrm{CD}^{+}{ }^{+} \mathrm{T}$ cells-released cytokine like IFN- $\gamma$ can efficiently suppress SIRT1 expression, and reshape the phenotype of monocytes. ${ }^{52}$ Considering the excellent effects of related compounds on inflammatory $\mathrm{T}$ cells (Figure 6), QLY could abrogate the inhibitory mechanism stated above, and consequently restored SIRT1 expression. But the direct effects of QLY on monocytes cannot be ignored too. Theoretically, the involvement of monocytes in AIA was prior to lymphocytes. ${ }^{28}$ Under such a context, the therapeutic effects of QLY on AIA cannot totally attribute to the impaired development of inflammatory lymphocytes. Furthermore, whether stimulus with certain compounds and their combination can simulate the situation in vivo is still questionable, since there are many other compounds existing in QLY-containing serum. Thus, total content of all the bioactive compounds may be more important.

Indeed, concentration is a vital variation deciding the final outcome, which is usually overlooked. Every single compound in QLY cannot achieve very high content in the blood after oral doses. Even for MT, the most abundant bioactive component, its concentration was lower than 100 $\mathrm{ng} / \mathrm{mL}$. Using serum concentration levels, SIN, SCA and MT can all suppress inflammatory $\mathrm{T}$ cell development. However, these stimuli barely affected the phenotype of AIA monocytes. These facts suggested that $\mathrm{T}$ cells were more sensitive to QLY treatment. When the concentrations were increased by 20-fold, these compounds showed antiinflammatory effects on AIA monocytes (Figure 7). We assume that repeated doses are necessary for QLY to treat monocytes-mediated inflammation in arthritic rats, which would result in chemical accumulation in the cells and tissues. This theory was supported by the observations stated above. Short-term QLY treatment did not decrease but further increased GM-CSF production in CIA rats (Figure 2C). It was also reflected by accelerated glycolysis during the early stages of QLY treatment (Figure 2F). However, the most plausible explanation is that many compounds derived from one plant share similar biosynthetic approaches and targets, and they achieve synergistic effects in decoction. There are many of such components identified in Figure 5. For example, KS-derived SCA and MT are all quinolizidine derivatives. Both compounds showed potent effects on immune targets. $^{24,26}$ Consequently, QLY-containing serum performed much better than pure compounds (Figure 4C-F).

\section{Conclusion}

The overall evidences demonstrate that QLY possesses notable immune regulatory properties, which serves as the foundation for its anti-rheumatic effects in vivo. On the one hand, QLY therapy impaired the maturation of $\mathrm{T}$ cells and their inflammatory differentiation. On the other hand, intensified stimulus of QLY impeded inflammatory polarization of monocytes. By simultaneously targeting inflammatory $\mathrm{T}$ cells and monocytes, QLY disrupted their immune 
feedback. It consequently reinforced immune consequences in CIA/AIA rats and further improved anti-rheumatic efficacy.

\section{Abbreviations}

MTX, methotrexate; CII, bovine type II collagen; IFA, incomplete Freund's adjuvant; PMA, phorbol 12myristate 13-acetate; BFA, brefeldin A; FBS, fetal bovine serum; HRP, horseradish peroxidase; PLS-DA, partial least squares-discrimination analysis; VIP, variable importance in projection; CFA, complete Freund's adjuvant; WB, western-blot; KEGG, Kyoto Encyclopedia of Genes and Genomes; GO, Gene Ontology; SCF, stem cell factor; PPP, pentose phosphate pathway; LPS, lipopolysaccharide; RA, rheumatoid arthritis; CIA, collagen-induced arthritis; QLY, QingLuo-Yin; AIA, adjuvant-induced arthritis; cDMARDs, conventional disease modifying anti-rheumatic drugs; bDMARDs, biological disease modifying antirheumatic drugs; WBCs, white blood cells; ELISA, enzyme linked immunosorbent assay; BCG, Bacillus Calmette-Guerin; WB, western-blot; SIN, sinomenine; MT, matrine; SCA, sophocarpine; GM-CSF, granulocyte-macrophage colony stimulating factor; TNF- $\alpha$, tumor necrosis factor alpha; IL-1 $\beta$, interleukin 1 beta; IL-17 $\alpha$, interleukin 17 alpha; IL-6, interleukin 6; SCF, stem cell factor; TCM, traditional Chinese medicine.

\section{Acknowledgment}

This work was supported by National Natural Science Foundation of China (81973828), Key Project of Natural Science Foundation of Anhui Province for College Scholar (KJ2019A0416 and KJ2020A0868), and Major Project of Natural Science Foundation of the Department of Education of Anhui province under (KJ2019ZD32).

\section{Author Contributions}

All authors made a significant contribution to conception and design, execution, acquisition of data, analysis and interpretation, or in all these areas; took part in drafting, revising or critically reviewing the article; gave final approval for the version to be published; have agreed on the journal to which the article has been submitted; and agree to be accountable for all aspects of the work.

\section{Disclosure}

The authors report no conflicts of interest.

\section{References}

1. Ponchel F, Vital EM, Kingsbury SR, El-Sherbiny Y. CD4 ${ }^{+}$T-cell subsets in rheumatoid arthritis. Int $J$ Cl Rheum. 2012;7(1):37-53. doi:10.2217/ijr.11.69

2. Thomas R, Turner M, Cope AP. High avidity autoreactive T cells with a low signalling capacity through the T-cell receptor: central to rheumatoid arthritis pathogenesis? Arthritis Res Ther. 2008;10 (4):210. doi:10.1186/ar2446

3. Scherer HU, Häupl T, Burmester GR. The etiology of rheumatoid arthritis. J Autoimmun. 2020;110:102400. doi:10.1016/j.jaut.2019.102400

4. Schulze-Koops H, Kalden JR. The balance of Th1/Th2 cytokines in rheumatoid arthritis. Best Pract Res Clin Rheumatol. 2001;15 (5):677-691. doi:10.1053/berh.2001.0187

5. Niu Q, Cai B, Huang ZC, Shi YY, Wang LL. Disturbed Th17/Treg balance in patients with rheumatoid arthritis. Rheumatol Int. 2012;32 (9):2731-2736. doi:10.1007/s00296-011-1984-x

6. Takeuchi T. Revolutionary change in rheumatoid arthritis management with biological therapy. Keio J Med. 2011;60(3):75-81. doi: $10.2302 / \mathrm{kjm} .60 .75$

7. Roberts CA, Dickinson AK, Taams LS. The interplay between monocytes/macrophages and $\mathrm{CD}^{+} \mathrm{T}$ cell subsets in rheumatoid arthritis. Front Immunol. 2015;6:571. doi:10.3389/fimmu.2015.00571

8. Kawanaka N, Yamamura M, Aita T, et al. CD14 ${ }^{+}, \mathrm{CD} 16^{+}$blood monocytes and joint inflammation in rheumatoid arthritis. Arthritis Rheumatol. 2010;46(10):2578-2586. doi:10.1002/art.10545

9. Rossol M, Kraus S, Pierer M, Baerwald C, Wagner U. The CD14 (bright) $\mathrm{CD} 16^{+}$monocyte subset is expanded in rheumatoid arthritis and promotes expansion of the Th17 cell population. Arthritis Rheum. 2012;64(3):671-677. doi:10.1002/art.33418

10. De-brito NM, Da-costa HC, Simões RL, Barja-Fidalgo C. Lipoxininduced phenotypic changes in $\mathrm{CD} 115^{+} \mathrm{LY} 6 \mathrm{C}^{\mathrm{hi}}$ monocytes tam precursors inhibits tumor development. Front Oncol. 2019;9:540. doi: $10.3389 /$ fonc. 2019.00540

11. Yrlid U, Jenkins CD, MacPherson GG. Relationships between distinct blood monocyte subsets and migrating intestinal lymph dendritic cells in vivo under steady-state conditions. J Immunol. 2006;176 (7):4155-4162. doi:10.4049/jimmunol.176.7.4155

12. Brühl H, Cihak J, Plachý J, et al. Targeting of Gr- $1^{+}, \mathrm{CCR} 2^{+}$monocytes in collagen-induced arthritis. Arthritis Rheum. 2007;56 (9):2975-2985. doi:10.1002/art.22854

13. Davignon JL, Hayder M, Baron M, et al. Targeting monocytes/macrophages in the treatment of rheumatoid arthritis. Rheumatology. 2013;52 (4):590-598. doi:10.1093/rheumatology/kes304

14. Hoes JN, Jacobs JWG, Buttgereit F, Bijlsma JWJ. Current view of glucocorticoid co-therapy with DMARDs in rheumatoid arthritis. Nat Rev Rheumatol. 2010;6(12):693-702. doi:10.1038/nrrheum.2010.179

15. Friedman B, Cronstein B. Methotrexate mechanism in treatment of rheumatoid arthritis. Joint Bone Spine. 2019;86(3):301-307. doi:10.1016/j.jbspin.2018.07.004

16. O'Dell JR, Mikuls TR, Taylor TH, et al. Therapies for active rheumatoid arthritis after methotrexate failure. $N$ Engl J Med. 2013;369 (4):307-318. doi:10.1056/NEJMoa1303006

17. Nam JL, Takase-Minegishi K, Ramiro S, et al. Efficacy of biological disease-modifying antirheumatic drugs: a systematic literature review informing the 2016 update of the EULAR recommendations for the management of rheumatoid arthritis. Ann Rheum Dis. 2017;76 (6):1108-1113. doi:10.1136/annrheumdis-2016-210713

18. An Y, Liu T, He D, et al. The usage of biological DMARDs and clinical remission of rheumatoid arthritis in China: a real-world large scale study. Clin Rheumatol. 2017;36(1):35-43. doi:10.1007/s10067-016-3424-5

19. Klareskog L, Gaubitz M, Rodriguez-Valverde V, et al. A long-term, open-label trial of the safety and efficacy of etanercept (Enbrel) in patients with rheumatoid arthritis not treated with other disease-modifying antirheumatic drugs. Ann Rheum Dis. 2006;65 (12):1578-1584. doi:10.1136/ard.2005.038349 
20. Tanaka Y, Hirata S, Saleem B, Emery P. Discontinuation of biologics in patients with rheumatoid arthritis. Clin Exp Rheumatol. 2013;31 (Suppl.78):S22-S27.

21. Singh JA, Cameron C, Noorbaloochi S, et al. Risk of serious infection in biological treatment of patients with rheumatoid arthritis: a systematic review and meta-analysis. Lancet. 2015;386 (9990):258-265. doi:10.1016/S0140-6736(14)61704-9

22. Zhang B, Wang X, Li S. An integrative platform of TCM network pharmacology and its application on a herbal formula, Qing-Luo-Yin. Evid Based Complement Alternat Med. 2013;2013:456747. doi:10.1155/2013/456747

23. Wu ZY. A Metabolomics Study of Traditional Chinese Medicine Recipe "Qing Luo Yin" in Treating Heat-Induced Paralysis Syndrome [master's dissertation]. Wuhu: Wannan Medical College; 2020:21-40.

24. Li X, Tang Z, Wen L, Jiang C, Feng Q. Matrine: a review of its pharmacology, pharmacokinetics, toxicity, clinical application and preparation researches. J Ethnopharmacol. 2020;269(1):113682. doi:10.1016/j.jep.2020.113682

25. Tang J, Raza A, Chen J, Xu H. A systematic review on the Sinomenine derivatives. Mini Rev Med Chem. 2018;18 (11):906-917. doi:10.2174/1389557517666171123212557

26. Zou F, Wang L, Liu H, et al. Sophocarpine suppresses NF$\kappa \mathrm{B}-$ mediated inflammation both in vitro and in vivo and inhibits diabetic cardiomyopathy. Front Pharmacol. 2019;10:1219. doi:10.3389/fphar.2019.01219

27. Wu M, Ma C, Wu Y, Li S. Simultaneous LC analysis of five bioactive alkaloids in an anti-angiogenesis herbal formula, Qing-Luo-Yin. Chromatographia. 2008;68(s7-s8):579-585. doi:10.1365/s10337008-0767-3

28. Billiau A, Matthys P. Collagen-induced arthritis and related animal models: how much of their pathogenesis is auto-immune, how much is auto-inflammatory? Cytokine Growth Factor Rev. 2011;22(56):339-344. doi:10.1016/j.cytogfr.2011.11.003

29. Furuya T, Salstrom JL, McCall-Vining S, et al. Genetic dissection of a rat model for rheumatoid arthritis: significant gender influences on autosomal modifier loci. Hum Mol Genet. 2000;9(15):2241-2250. doi:10.1093/oxfordjournals.hmg.a018915

30. Zuo J, Yin Q, Wang YW, et al. Inhibition of NF- $\mathrm{kB}$ pathway in fibroblastlike synoviocytes by $\alpha$-mangostin implicated in protective effects on joints in rats suffering from adjuvant-induced arthritis. Int Immunopharmacol. 2018;56:78-89. doi:10.1016/j.intimp.2018.01.016

31. Psychogios N, Hau DD, Peng J, et al. The human serum metabolome. PLoS One. 2011;6(2):e16957. doi:10.1371/journal.pone.0016957

32. Pérez-Enciso M, Tenenhaus M. Prediction of clinical outcome with microarray data: a partial least squares discriminant analysis (PLS-DA) approach. Hum Genet. 2003;112(5-6):581-592. doi:10.1007/s00439-003-0921-9

33. Smoot ME, Ono K, Ruscheinski J, Wang PL, Ideker T. Cytoscape 2.8: new features for data integration and network visualization. Bioinformatics. 2011;27(3):431-432. doi:10.1093/bioinformatics/ btq 675

34. Ma HL, Liang S, Li J, et al. IL-22 is required for Th17 cell-mediated pathology in a mouse model of psoriasis-like skin inflammation. J Clin Invest. 2008;118(2):597-607. doi:10.1172/JCI33263

35. Daniela F. New Insights Into the Molecular and Cellular Requirements of Lymphocyte Development [doctoral thesis]. Basel: University of Basel, Faculty of Science; 2016:32-106.

36. Sierra-Filardi E, Puig-Krger A, Blanco FJ, et al. Activin A skews macrophage polarization by promoting a proinflammatory phenotype and inhibiting the acquisition of anti-inflammatory macrophage markers. Blood. 2011;117(19):5092-5101. doi:10.1182/blood-201009-306993
37. Vishnyakova P, Poltavets A, Karpulevich E, et al. The response of two polar monocyte subsets to inflammation. Biomed Pharmacother. 2021;139(327):111614. doi:10.1016/j.biopha.2021.111614

38. Rodgers LC, Cole J, Rattigan KM, et al. The rheumatoid synovial environment alters fatty acid metabolism in human monocytes and enhances CCL20 secretion. Rheumatology. 2020;59(4):869-878. doi:10.1093/rheumatology/kez378

39. Lotfi N, Zhang GX, Esmaeil N, Rostami A. Evaluation of the effect of GM-CSF blocking on the phenotype and function of human monocytes. Sci Rep-UK. 2020;10(1):1567. doi:10.1038/s41598-02058131-2

40. Larysa BB, Valentina MK. Pyrazinamide effects on cartilage type II collagen amino acid composition. Int J Pept. 2012;2012:781785. doi: $10.1155 / 2012 / 781785$

41. Rhoads JP, Major AS, Rathmell JC. Fine tuning of immunometabolism for the treatment of rheumatic diseases. Nat Rev Rheumatol. 2017;13(5):313-320. doi:10.1038/nrrheum.2017.54

42. Wang R, Li DF, Hu YF, et al. Qing-Luo-Yin alleviated monocytes/ macrophages-mediated inflammation in rats with adjuvant-induced arthritis by disrupting their interaction with (pre)-adipocytes through PPAR- $\gamma$ signaling. Drug Des Devel Ther. 2021;15:3105-3118. doi:10.2147/DDDT.S320599

43. Kiefer J, Zeller J, Bogner B, et al. An unbiased flow cytometry-based approach to assess subset-specific circulating monocyte activation and cytokine profile in whole blood. Front Immunol. 2021;12:641224. doi:10.3389/fimmu.2021.641224

44. Jia Y, Han S, Li J, et al. IRF8 is the target of SIRT1 for the inflammation response in macrophages. Innate Immun. 2017;23 (2):188-195. doi:10.1177/1753425916683751

45. Zhang M, Zhou L, Xu Y, et al. A STAT3 palmitoylation cycle promotes TH17 differentiation and colitis. Nature. 2020;586 (7829):434-439. doi:10.1038/s41586-020-2799-2

46. Li S, Lu AP, Wang YY, Li YD. Suppressive effects of a Chinese herbal medicine Qing-Luo-Yin extract on the angiogenesis of collagen-induced arthritis in rats. Am J Chin Med. 2003;31 (5):713-720. doi:10.1142/S0192415X03001430

47. Guo W. Comparative Study On the Effect of Anti-Synovial Angiogenesis of Qingluoyin and Wenluoyin in Rheumatoid Arthritis [master's dissertation] Beijing: China Academy of Chinese Medical Sciences; 2011:40-70.

48. Jiang TT, Ji CF, Cheng XP, et al. $\alpha$-Mangostin alleviated HIF-1 $\alpha$ mediated angiogenesis in rats with adjuvant-induced arthritis by suppressing aerobic glycolysis. Front Pharmacol. 2021. doi:10.3389/fphar.2021.785586

49. Zuo J, Wang X, Liu Y, et al. Integrating network pharmacology and metabolomics study on anti-rheumatic mechanisms and antagonistic effects against methotrexate-induced toxicity of Qing-Luo-Yin. Front Pharmacol. 2018;2018:01472. doi:10.3389/fphar.2018.01472

50. Phillips JL, Azari P. Zinc transferrin. Enhancement of nucleic acid synthesis in phytohemagglutinin-stimulated human lymphocytes. Cell Immunol. 1974;10(1):31-37. doi:10.1016/0008-8749(74)90148-8

51. Wang QH, Li Y, Dou DY, et al. Nicotinamide mononucleotide-elicited NAMPT signaling activation aggravated adjuvant-induced arthritis in rats by affecting peripheral immune cells differentiation. Int Immunopharmacol. 2021;98:107856. doi:10.1016/j.intimp.2021.107856

52. Li P, Zhao Y, Wu X, et al. Interferon gamma (IFN- $\gamma$ ) disrupts energy expenditure and metabolic homeostasis by suppressing SIRT1 transcription. Nucleic Acids Res. 2012;40(4):1609-1620. doi:10.1093/ nar/gkr984 


\section{Publish your work in this journal}

The Journal of Inflammation Research is an international, peerreviewed open-access journal that welcomes laboratory and clinical findings on the molecular basis, cell biology and pharmacology of inflammation including original research, reviews, symposium reports, hypothesis formation and commentaries on: acute/chronic inflammation; mediators of inflammation; cellular processes; molecular mechanisms; pharmacology and novel anti-inflammatory drugs; clinical conditions involving inflammation. The manuscript management system is completely online and includes a very quick and fair peerreview system. Visit http://www.dovepress.com/testimonials.php to read real quotes from published authors.

Submit your manuscript here: https://www.dovepress.com/journal-of-inflammation-research-journal 